\title{
Inhibition of Advanced Glycation End Products Formation Attenuates Cardiac Electrical and Mechanical Remodeling and Vulnerability to Tachyarrhythmias in Diabetic Rats $\$$
}

\author{
(1) Gwo-Jyh Chang, Yung-Hsin Yeh, Wei-Jan Chen, Yu-Shien Ko, Jong-Hwei S. Pang, \\ and Hsiao-Yu Lee \\ Graduate Institute of Clinical Medicinal Sciences, College of Medicine, Chang Gung University (G.-J.C., J.-H.S.P., H.-Y.L.), and \\ Cardiovascular Medicine, Chang Gung Memorial Hospital (G.-J.C., Y.-H.Y., W.-J.C., Y.-S.K.), Tao-Yuan, Taiwan
}

Received July 18, 2018; accepted October 23, 2018

\begin{abstract}
Diabetic patients with cardiomyopathy show a higher incidence of arrhythmias and sudden death. Chronic hyperglycemia induces the formation of advanced glycation end products (AGEs), which contribute to the pathogenesis of diabetic cardiomyopathy. This study investigated whether inhibition of AGEs formation by aminoguanidine (AG) could prevent cardiac electromechanical and arrhythmogenic remodeling in diabetes mellitus. Streptozotocininduced diabetic rats received AG (100 mg/kg daily, i.p.) or vehicle (normal saline, i.p.) for 5 weeks. The rats underwent hemodynamic recording to evaluate cardiac function, and heart preparations were used to determine the electrical, mechanical, and biochemical functions. In vitro high glucose-induced AGEs formation, reactive oxygen species (ROS) generation, and action potential changes were examined in $\mathrm{HL}-1$ atrial cells. AG treatment improved the diabetes-induced depression in left ventricular pressure and the
\end{abstract}

relaxation rate, and normalized the prolongation of QTc intervals in anesthetized rats. AG reduced the vulnerabilities to atrial and ventricular tachyarrhythmias in perfused diabetic hearts. AG normalized the prolonged action potential duration in diabetic atrial and ventricular muscles, which was correlated with the restoration of both transient outward $\left(I_{\text {to }}\right)$ and steady-state outward $\left(I_{\mathrm{ss}}\right) \mathrm{K}^{+}$current densities in cardiomyocytes. The abnormal kinetics of $\mathrm{Ca}^{2+}$ transients and contraction were reversed in cardiomyocytes from AG-treated diabetic rats, along with parallel preservation of sarco(endo)plasmic reticulum $\mathrm{Ca}^{2+}$-ATPase (SERCA2a) expression. Furthermore, ex vivo and in vitro studies showed AG attenuated AGEs and ROS formation. Thus, long-term administration of AG ameliorated cardiac electromechanical remodeling and arrhythmogenicity in diabetic rats and may present an effective strategy for the prevention of diabetes-associated arrhythmias.

\section{Introduction}

Diabetes mellitus (DM) is associated with an increased risk of cardiovascular morbidity and mortality, much of which stems from cardiomyopathy, a unique disease that can progress toward overt heart failure independent of vascular complications (Bugger and Abel, 2014). Diabetic cardiomyopathy, which can occur in both type 1 and type 2 diabetes even with good glycemic control, is characterized by early-onset diastolic and late-onset systolic dysfunction, as well as ECG abnormalities such as QT prolongation and dispersion (Veglio et al., 2004; Huynh et al., 2014). Such ECG changes may

This work was supported by the Chang Gung Medical Research Foundation [CMRPD170501 3 and BMRP468]; and the Ministry of Science and Technology of Taiwan [NSC96-2320-B-182-026-MY3 and NSC99-2320-B-182-005MY3].

https://doi.org/10.1124/jpet.118.252080.

S This article has supplemental material available at jpet.aspetjournals.org. increase the incidence of life-threatening ventricular arrhythmias and sudden death (Veglio et al., 2004). Diabetes is also associated with an increased incidence of atrial fibrillation (Movahed et al., 2005). There is emerging evidence to suggest that defects in intracellular $\mathrm{Ca}^{2+}$ homeostasis can contribute to myocardial mechanical and electrical dysfunction (Bugger and Abel, 2014). As a consequence, agents that restore the normal electrical and $\mathrm{Ca}^{2+}$ handling properties may represent a useful approach for the prevention of these lethal complications.

Diabetes triggers a number of signaling pathways that lead to the development of cardiomyopathy (Bugger and Abel, 2014). Chronic hyperglycemia is associated with the formation of advanced glycation end products (AGEs), which are biochemical end products of nonenzymatic glycosylation that contribute to diabetic complications (Norton et al., 1996; Brownlee, 2001). Growing evidence suggests that targeting the accumulation of AGE may be a potential strategy for

ABBREVIATIONS: AERP, atrial effective refractory period; AG, aminoguanidine; AGE, advanced glycation end product; $A \mathrm{AD}_{25}, 50,90$, action potential duration measured at $25 \%, 50 \%$, and $90 \%$ repolarization; AT, atrial tachyarrhythmias; BW, body weight; DM, diabetes mellitus; $\mathrm{d} P / \mathrm{d} t_{\text {max }}$ and $\mathrm{d} P / \mathrm{d} t_{\min }$, maximal rate of rise and fall of LV pressure; ECG, electrocardiogram; HG, high glucose; HW, heart weight; IACT, interatrial conduction time; $I_{\mathrm{Ca}, \mathrm{L}}$, L-type $\mathrm{Ca}^{2+}$ current; $I_{\mathrm{K} 1}$, inward rectifier $\mathrm{K}^{+}$current; $I_{\mathrm{SS}}$, steady-state outward $\mathrm{K}^{+}$current; $I_{\text {to }}$, transient outward $\mathrm{K}^{+}$current; $\mathrm{LV}$, left ventricle; LVW, LV weight; NCX, $\mathrm{Na}^{+}-\mathrm{Ca}^{2+}$ exchanger; NG, normal glucose; PLB, phospholamban; RAGE, AGE receptor; ROS, reactive oxygen species; RyR2, ryanodine receptor $\mathrm{Ca}^{2+}$ release channel; SERCA, sarco(endo)plasmic reticulum $\mathrm{Ca}^{2+}$-ATPase; siRNA, small interfering RNA; STZ, streptozotocin; VERP, ventricular effective refractory period; $V_{\max }$, maximal upstroke velocity of action potential. 
TABLE 1

General characteristics, hemodynamic and electrocardiogram variables in control and diabetic rats treated with vehicle or aminoguanidine

Data are expressed as mean \pm S.E.M. "Initial" and "final" represent the observation times of the beginning of week 1 and the end of week 6 of the experimental period, respectively. Rate-corrected QT intervals (QTc) were derived using the Bazett's formula QTc $(\mathrm{ms})=$ measured QT interval $(\mathrm{ms}) /[$ square root of RR interval (s)].

\begin{tabular}{|c|c|c|c|c|}
\hline & Con + Vehicle & DM + Vehicle & $\mathrm{Con}+\mathrm{AG}$ & $\mathrm{DM}+\mathrm{AG}$ \\
\hline Sample size $(n)$ & 16 & 18 & 15 & 22 \\
\hline Initial BW (g) & $211.9 \pm 5.2$ & $205.8 \pm 5.1$ & $208.7 \pm 4.2$ & $209.8 \pm 3.4$ \\
\hline Final BW (g) & $380.3 \pm 14.1$ & $216.1 \pm 8.6^{a}$ & $384.3 \pm 13.4$ & $226.8 \pm 9.7^{a}$ \\
\hline HW (g) & $1.33 \pm 0.06$ & $0.93 \pm 0.04^{a}$ & $1.38 \pm 0.05$ & $0.90 \pm 0.04^{a}$ \\
\hline AW (g) & $0.17 \pm 0.01$ & $0.15 \pm 0.01$ & $0.18 \pm 0.01$ & $0.14 \pm 0.01$ \\
\hline LVW (g) & $0.67 \pm 0.03$ & $0.45 \pm 0.02^{a}$ & $0.66 \pm 0.02$ & $0.42 \pm 0.02^{a}$ \\
\hline HW/BW (mg/g) & $3.49 \pm 0.08$ & $4.34 \pm 0.12^{a}$ & $3.62 \pm 0.11$ & $3.98 \pm 0.08^{b, c}$ \\
\hline AW/BW (mg/g) & $0.44 \pm 0.01$ & $0.70 \pm 0.03^{a}$ & $0.47 \pm 0.02$ & $0.61 \pm 0.03^{a, c}$ \\
\hline LVW/BW (mg/g) & $1.76 \pm 0.06$ & $2.11 \pm 0.08^{b}$ & $1.73 \pm 0.07$ & $1.87 \pm 0.04^{c}$ \\
\hline Blood glucose (mg/dl) & $164.1 \pm 12.1$ & $455.6 \pm 19.3^{a}$ & $152.0 \pm 16.2$ & $399.0 \pm 25.3^{a}$ \\
\hline Sample size $(n)$ & 24 & 27 & 24 & 26 \\
\hline HR (beats/min) & $325 \pm 12$ & $265 \pm 9^{a}$ & $324 \pm 11$ & $274 \pm 7^{b}$ \\
\hline MAP (mm Hg) & $70.4 \pm 2.4$ & $71.2 \pm 3.2$ & $70.2 \pm 2.3$ & $72.2 \pm 3.0$ \\
\hline $\operatorname{LVESP}(\mathrm{mm} \mathrm{Hg})$ & $118.1 \pm 2.7$ & $99.1 \pm 2.1^{a}$ & $118.5 \pm 2.2$ & $107.9 \pm 2.2^{c, d}$ \\
\hline LVEDP (mm Hg) & $4.6 \pm 0.7$ & $7.1 \pm 0.6^{d}$ & $5.2 \pm 0.6$ & $5.7 \pm 0.7$ \\
\hline LVDP (mm Hg) & $113.5 \pm 2.7$ & $92.3 \pm 2.5^{a}$ & $113.2 \pm 2.3$ & $102.2 \pm 2.1^{b, c}$ \\
\hline $\mathrm{d} P / \mathrm{d} t_{\max }(\mathrm{mm} \mathrm{Hg} / \mathrm{s})$ & $7445 \pm 461$ & $5782 \pm 395^{d}$ & $8045 \pm 409$ & $6608 \pm 357$ \\
\hline $\mathrm{d} P / \mathrm{d} t_{\min }(\mathrm{mm} \mathrm{Hg} / \mathrm{s})$ & $5573 \pm 363$ & $3939 \pm 256^{a}$ & $5075 \pm 277$ & $4606 \pm 191$ \\
\hline Tau (ms) & $11.1 \pm 0.7$ & $16.3 \pm 0.4^{a}$ & $11.8 \pm 0.8$ & $13.7 \pm 0.4^{d, e}$ \\
\hline $\mathrm{PR}(\mathrm{ms})$ & $60.6 \pm 2.0$ & $59.9 \pm 2.1$ & $61.2 \pm 1.7$ & $59.3 \pm 1.6$ \\
\hline $\mathrm{QRS}(\mathrm{ms})$ & $15.3 \pm 0.4$ & $15.3 \pm 0.5$ & $15.0 \pm 0.3$ & $14.8 \pm 0.3$ \\
\hline QTc $(\mathrm{ms})$ & $214.8 \pm 4.5$ & $253.4 \pm 10.2^{b}$ & $212.7 \pm 5.2$ & $224.6 \pm 4.2^{c}$ \\
\hline
\end{tabular}

AG, aminoguanidine; AW, atrial weight; BW; body weight; Con, control; DM, diabetes mellitus; $\mathrm{d} P / \mathrm{d} t_{\max }$ and $\mathrm{d} P / \mathrm{d} t_{\min }$ maximal rate of rise and fall of LV pressure; HR, heart rate; HW, heart weight; LVDP, LV developed pressure; LVEDP

LV end-diastolic pressure; LVESP, LV end-systolic pressure; LVW, left ventricular weight; MAP, mean arterial pressure; Tau, time constant of LV pressure decay.

${ }^{a} P<0.001$ vs. Con + vehicle group.

${ }^{b} P<0.01$ vs. Con + vehicle group.

${ }^{c} P<0.05$ vs. $\mathrm{DM}+$ vehicle group.

${ }^{d} P<0.05$ vs. Con + vehicle.

${ }^{e} P<0.01$ vs. DM + vehicle group.

preventing cardiomyopathy (Yamagishi et al., 2008; Bodiga et al., 2014). AGEs cause damage to cells, possibly by means of protein modification or interactions with AGE receptors (RAGEs), generating reactive oxygen species (Brownlee, 2001; Bodiga et al., 2014). Oxidative stress plays a crucial role in the pathogenesis of diabetic complications (Huynh et al., 2014) and is a known major modulator of cardiac $\mathrm{K}^{+}$channels. Under diabetic conditions, the transient outward $\mathrm{K}^{+}$current $\left(I_{\mathrm{to}}\right)$ density in cardiac cells is down-regulated by oxidative stress (Li et al., 2005) and is augmented after relief of this stress. Modulation of redox signaling is thus an attractive strategy for preventing diabetic cardiac remodeling (Huynh et al., 2014).

Aminoguanidine (AG), a nucleophilic hydrazine compound, is the prototype and most extensively studied inhibitor of AGEs formation (Brownlee et al., 1986). AG inhibits AGEs formation via trapping of reactive carbonyl intermediates and hence preventing modification of nucleophilic residues in proteins. AG also acts as an inhibitor of inducible NO synthase (Corbett et al., 1992) and as an antioxidant (Giardino et al., 1998). AG-mediated inhibition of the cross-linking of AGEs on collagen molecules has been shown to retard diabetesassociated myocardial stiffness and the decrease in myocardial compliance (Norton et al., 1996). AG therapy has been reported to prevent diabetic cardiac hypertrophy by blocking protein carbonylation (Stadler et al., 2005), and to attenuate contractile dysfunction either by inhibiting the accumulation of AGEs in the arterial wall (Wu et al., 2008) or by decreasing AGEs-induced microtubule stabilization in the myocardium (Yuan et al., 2015). Although these results suggest a potential role for the AGEs inhibitor for prevention of diabetic cardiovascular complications, the effect of this intervention on diabetes-induced cardiac electromechanical and arrhythmogenic remodeling remains unknown. In this study, we comprehensively investigated the effects of long-term AG treatment on the status of cardiac mechanical function and associated $\mathrm{Ca}^{2+}$ handling, as well as electrophysiologic and arrhythmogenic properties in streptozotocin (STZ)-induced diabetic rats.

\section{Materials and Methods}

Animal Preparation. All experiments were approved by the Institutional Animal Care and Use Committee of Chang Gung University and were performed in accordance with the Guide for the Care and Use of Laboratory Animals (National Research Council, 2011). Male Sprague-Dawley rats (BioLASCO Co., Taipei, Taiwan) weighing 190-220 g, aged 6 weeks, received a single tail vein injection of STZ ( $65 \mathrm{mg} / \mathrm{kg}$ ). STZ was freshly dissolved in $0.9 \%$ saline containing $0.1 \mathrm{M}$ sodium citrate, $\mathrm{pH}$ adjusted to 4.5. One week after STZ injection, animals that had at least a 3-fold increase in fasting plasma blood glucose levels compared with preinjection levels were classified as diabetic. The blood glucose level was determined by using a digital glucometer and test strips (Ascensia ELITE; Bayer HealthCare AG, Leverkusen, Germany). Rats injected with comparable volumes of citrate-buffered saline served as nondiabetic controls. Diabetic and control rats were received either AG ( $100 \mathrm{mg} / \mathrm{kg}$ daily, i.p.) or vehicle (saline, i.p.), respectively, for 5 weeks in an identical fashion. Rats were housed in a 12-hour light/dark cycle animal room and were allowed free access to standard chow and water.

Hemodynamic and Electrocardiogram Measurements. After the 6-week experimental period, the rats were anesthetized with 

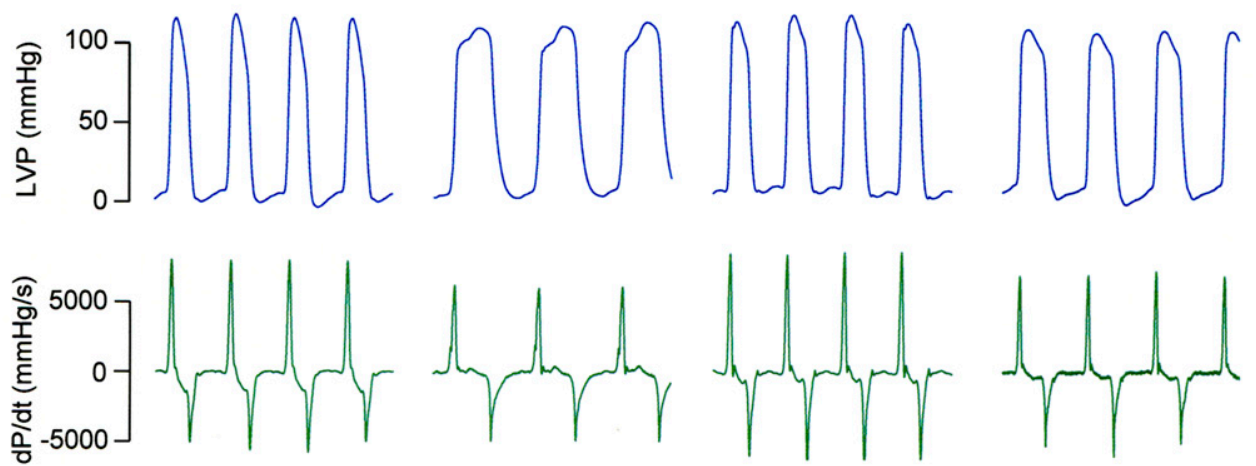

Fig. 1. In vivo LV performance and ECG recordings in anesthetized rats and ex vivo conduction electrogram recordings in isolated hearts. (A) Representative traces of LV pressure (LVP), the first derivative of $\operatorname{LVP}(\mathrm{d} P / \mathrm{d} t)$, and lead II ECG recorded from control (Con) and diabetic (DM) rats with vehicle (Veh) or AG treatment. (B) Representative His bundle (HBE) and ventricular (VE) electrograms of Langendorff-perfused hearts from various groups. Electrograms were recorded at an atrial pacing cycle length of 200 milliseconds. A, atrial depolarization; $\mathrm{H}$, His

B
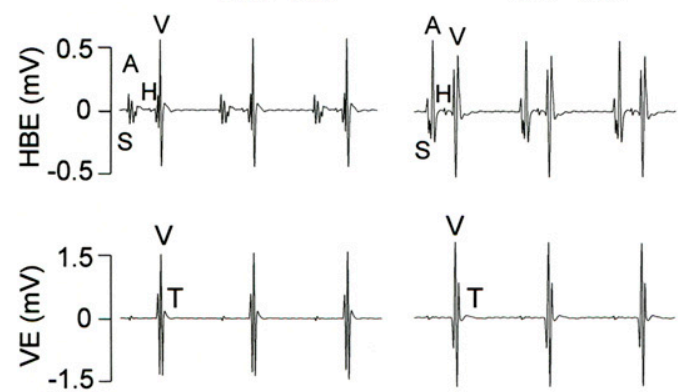<smiles>CC(C)(C)C(C)(C)C(C)(C)C(C)(C)C(C)(C)C</smiles>

Con+AG
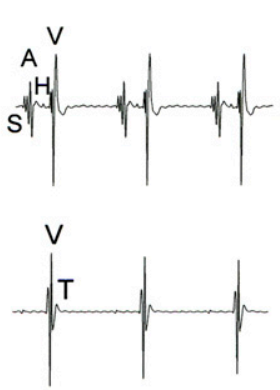

$\mathrm{DM}+\mathrm{AG}$
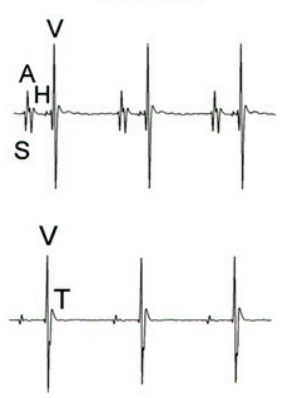
tifact; T, ventricular repolarization; V, ventricular depolarization. urethane (1.25 g/kg, i.p.). Blood pressure was measured by inserting a polyethylene cannula into the femoral artery and connecting it to a pressure transducer with a bridge amplifier. Lead II electrocardiograms (ECGs) were recorded by a biologic amplifier. A 1.9F microtip catheter (Scisense, London, ON, Canada) connected to a pressure-volume conductance system (896B; Scisense) was used to measure left ventricular (LV) pressure. Output signals from these amplifiers were connected to an ACQ16 acquisition system (DSI Ponemah, Valley View, OH) and were computed using an analysis program (P3 Plus 4.80-SP4; DSI Ponemah).

Histologic Analysis. The atrial and ventricular sections $(5 \mu \mathrm{m}$ thickness) were stained with Masson's trichrome and viewed under a light microscope $(200 \times$ amplification; Axio Observer Z1; Carl Zeiss Light Microscopy, Göttingen, Germany). The fraction of interstitial fibrosis area was quantified on five fields per slice for each heart using Image-Pro Premier 9.0 (Media Cybernetics, Rockville, MD).

Electrophysiological Studies in Perfused Hearts. Rats were sacrificed after the completion of hemodynamic recordings. The heart was quickly excised, mounted on a Langendorff perfusion apparatus, and retrogradely perfused at a rate of $6 \mathrm{ml} / \mathrm{min}$ per (gram cardiac tissue) with oxygenated $\left(95 \% \mathrm{O}_{2}\right.$ and $\left.5 \% \mathrm{CO}_{2}\right)$ normal Tyrode's solution (composition [millimolar]: $137 \mathrm{NaCl}, 5.4 \mathrm{KCl}, 1.1 \mathrm{MgCl}_{2}, 11.9 \mathrm{NaHCO}_{3}$, $0.33 \mathrm{NaH}_{2} \mathrm{PO}_{4}, 1.8 \mathrm{CaCl}_{2}$ and 11 dextrose) at $37^{\circ} \mathrm{C}$ as described elsewhere (Chang et al., 2014). A 1.2F octapolar electrophysiology catheter (Scisense) was used to record the His bundle electrogram, and the other platinum electrode was used to record epicardial ventricular signals.

To pace the atrium and ventricle, one pacing electrode was placed on the right atrium, and the second was placed on the right ventricular apex. Pacing stimuli (1-millisecond duration, twice-threshold voltage) were delivered by a programmable stimulator (DTU 215; Fischer Imaging, Denver, CO). The signals were continuously recorded on a chart recorder (WindowGraf; Gould, Cleveland, $\mathrm{OH}$ ) and digitized simultaneously with a data acquisition system (IWX/214; iWorx, Dover, NH). The right atrium was then paced at a constant rate which was slightly faster than the spontaneous heart rate. At this constant rate of pacing, the intra-atrial, AV nodal, and His-ventricular conduction intervals, and QT interval were measured.

Incremental right atrial pacing was used to determine the Wenckebach cycle length, at which the 1:1 AV conduction was lost. Atrial premature extrastimulation $\left(\mathrm{S}_{2}\right)$ after a train of constant rate atrial pacing $\left(\mathrm{S}_{1} \mathrm{~S}_{1}\right)$ for eight beats was then performed to obtain the refractory periods of the atria, AV node, and His-Purkinje system. The ventricular effective refractory period (VERP) was determined using a ventricular extrastimulation study protocol.

Atrial tachyarrhythmia was induced with 1-second burst pacing (a train of 100 square wave pulses, 1 millisecond in duration at a frequency of $100 \mathrm{~Hz}$ ) after eight regularly paced stimuli $\left(\mathrm{S}_{1}\right)$ at 200-millisecond pacing cycle length and $5 \times$ diastolic threshold. Ventricular tachyarrhythmia was evoked by programmed electrical extrastimulation. An arrhythmia scoring system was used as described in the Supplemental Material.

Electrical Measurements on Cardiac Muscles. The left atrial strips or LV papillary muscles were superfused at a rate of $20 \mathrm{ml} / \mathrm{min}$ with oxygenated normal Tyrode's solution, and were stimulated with $1.5 \times$ threshold strength pulses (pulse duration: 1 millisecond) through platinum field electrodes (Chang et al., 2014). Transmembrane 
TABLE 2

Conduction system parameters recorded from the isolated perfused hearts of control and diabetic rats treated with vehicle or aminoguanidine

Data (in milliseconds) are expressed as mean \pm S.E.M. The QT interval (interval between $\mathrm{V}$ and $\mathrm{T}$ wave) was used as the parameter for monitoring ventricular repolarization. SA, AH, HV, and QT intervals were recorded at a pacing cycle length of $200 \mathrm{~ms}$.

\begin{tabular}{lrrrr}
\hline & $\begin{array}{c}\text { Con + Vehicle } \\
(n=21)\end{array}$ & $\begin{array}{c}\text { DM + Vehicle } \\
(n=24)\end{array}$ & \multicolumn{1}{c}{$\begin{array}{c}\text { Con }+ \text { AG } \\
(n=21)\end{array}$} & \multicolumn{1}{c}{$\begin{array}{c}\text { DM }+ \text { AG } \\
(n=24)\end{array}$} \\
\hline BCL & $243.3 \pm 5.9$ & $269.2 \pm 8.6^{a}$ & $241.4 \pm 6.6$ & $255.6 \pm 5.6$ \\
SA & $10.5 \pm 0.3$ & $10.9 \pm 0.4$ & $9.9 \pm 0.4$ & $10.5 \pm 0.4$ \\
AH & $25.7 \pm 1.0$ & $25.0 \pm 0.7$ & $23.7 \pm 0.8$ & $26.1 \pm 1.0$ \\
HV & $19.5 \pm 0.8$ & $18.6 \pm 1.0$ & $18.9 \pm 0.5$ & $19.5 \pm 0.8$ \\
QT & $42.1 \pm 3.5$ & $54.0 \pm 3.0^{a}$ & $43.3 \pm 2.4$ & $45.0 \pm 2.1^{b}$ \\
WCL & $114.8 \pm 3.3$ & $123.8 \pm 3.3$ & $113.3 \pm 2.6$ & $118.8 \pm 2.2$ \\
AERP & $38.6 \pm 1.7$ & $48.8 \pm 2.0^{c}$ & $40.0 \pm 2.7$ & $41.3 \pm 1.5^{b}$ \\
AVNERP & $88.6 \pm 2.6$ & $103.8 \pm 2.8^{d}$ & $90.5 \pm 2.6$ & $96.7 \pm 2.0^{a}$ \\
HPFRP & $117.6 \pm 2.4$ & $134.2 \pm 2.7^{d}$ & $121.0 \pm 4.0$ & $126.9 \pm 1.7^{c}$ \\
VERP & $40.0 \pm 4.1$ & $58.8 \pm 3.1^{c}$ & $44.8 \pm 3.8$ & $46.7 \pm 2.2^{b}$ \\
\hline
\end{tabular}

AERP, atrial effective refractory period; AG, aminoguanidine; $\mathrm{AH}$, atrio-His bundle conduction interval; AVNERP, AV nodal effective refractory period; BCL, basic cycle length; Con, control; DM, diabetes mellitus; HPFRP, His-Purkinje system functional refractory period; HV, His-ventricular conduction interval; SA, stimulusatrial conduction interval; VERP, ventricular effective refractory period; WCL, Wenckebach cycle length.

${ }^{a} P<0.05$ vs. Con + vehicle group.

${ }^{b} P<0.05$ vs. $\mathrm{DM}+$ vehicle group

${ }^{c} P<0.01$ vs. Con + vehicle group.

${ }^{d} P<0.001$ vs. Con + vehicle group.

potentials were recorded using an Axoclamp 2B amplifier (Molecular Devices, Sunnyvale, CA).

Measurements of $\mathrm{Ca}^{2+}$ Transient and Cell Shortening. Single cardiomyocytes were isolated using a modification of the enzymatic dissociation procedure previously described elsewhere (Chang et al., 2014; for details, see the Supplementary Materials and Methods). Intracellular $\mathrm{Ca}^{2+}$ transients were measured by fura-2 fluorescence at room temperature $\left(25-27^{\circ} \mathrm{C}\right)$ as described previously elsewhere (Chang et al., 2014). Cytosolic loading of fura-2 was achieved by incubating cardiomyocytes with $5 \mu \mathrm{M}$ fura-2-AM and $2 \%$ Pluronic F-127 for 30 minutes at room temperature.

After we had washed out the excess fura-2-AM, the loaded myocytes were transferred to $1.8 \mathrm{mM} \mathrm{Ca}^{2+}$-containing buffer and were stimulated by 2 -millisecond square wave pulses with a voltage approximately 30\%-40\% above the threshold, at a frequency of $1 \mathrm{~Hz}$. The cells were illuminated at a wavelength of 340 or $380 \mathrm{~nm}$ using a RatioMaster fluorometer system (PTI; HORIBA Scientific, Edison, NJ). The ratio of the fluorescence signal $\left(\mathrm{F}_{340} / \mathrm{F}_{380}\right)$ was used as the indicator of intracellular $\mathrm{Ca}^{2+}$ concentration. Cell shortening was measured simultaneously with a video edge-detection system (Crescent Electronics, Sandy, UT). Caffeine-induced $\mathrm{Ca}^{2+}$ transients were evoked by rapidly bath application of $10 \mathrm{mM}$ caffeine 10 seconds after stopping field stimulations of the myocytes with electrical pulses (for 20 seconds at $1-\mathrm{Hz}$ frequency) to ensure a stable $\mathrm{SR} \mathrm{Ca}^{2+}$ load.

Western Blot Analysis. Western blotting was performed to measure the expression of various proteins in LV samples or HL-1 cells using primary antibodies against ryanodine receptor $\mathrm{Ca}^{2+}$ release channel (RyR2) (Thermo Fisher Scientific, Waltham, MA), sarco(endo)plasmic reticulum $\mathrm{Ca}^{2+}$-ATPase 2a (SERCA2a) (Badrilla, Leeds, United Kingdom), phospholamban (PLB) (Badrilla), phosphorylated pSer16-PLB (Millipore Bioscience Research Reagents, Temecula, CA), $\mathrm{Na}^{+} / \mathrm{Ca}^{2+}$ exchanger (NCX1) (Santa Cruz Biotechnology, Dallas, TX), AGE (TransGenic, Fukuoka, Japan), or RAGE (Abcam, Cambridge, MA). The blot probed with GAPDH (Santa Cruz Biotechnology) or tubulin antibody (Santa Cruz Biotechnology) was used as an internal control. Details are described in the Supplemental Material.

Detection of NADPH-Dependent Superoxide Production by Chemiluminescence. Generation of superoxide was estimated in fresh LV tissue (approximately $2 \mathrm{mg}$ ) with lucigenin-enhanced chemiluminescence using a luminometer (Plate CHAMELEON; Hidex, Turku, Finland) as described elsewhere (Yeh et al., 2016). Photon outputs were measured after addition of lucigenin $(5 \mu \mathrm{M})$ and NADPH $(100 \mu \mathrm{M})$. Chemiluminescence data were recorded from five tissues for each heart and normalized to the dry weight of tissues.

In Vitro Transfection of Small Interfering RNA. HL-1 atrial myocytes in four-well dishes were transfected with chemically synthesized small interfering RNAs (siRNAs) for RAGE, or its control siRNA ( $200 \mathrm{nM} /$ well; Dharmacon, Lafayette, CO) for 48 hours before cell treatment using DharmaFECT 1 (Dharmacon) according to the manufacturer's instructions (Yeh et al., 2016). The on-target siRNAsmart pool included four sequences.

Detection of Intracellular Reactive Oxygen Species. Intracellular reactive oxygen species (ROS) generation in HL-1 cells was measured using cell-permeable fluorescent dyes (2,7-dichlorodihydrofluorescein diacetate or dihydroethidium) at a concentration of $10 \mu \mathrm{M}$ (Yeh et al., 2016). The ROS-mediated fluorescence was observed under a confocal microscope (TCS SP2; Leica, Wetzlar, Germany) with excitation using a 488-nm argon laser. Emission was recorded using a long-pass $>530$-nm filter set to acquire two-dimensional images $(512 \times 512$ pixels $)$.

Whole-Cell Patch-Clamp Recording. Ionic currents were recorded in $\mathrm{LV}$ or atrial myocytes at room temperature $\left(25-27^{\circ} \mathrm{C}\right)$ using the whole-cell patch-clamp configuration (pipette resistance of 2-5 $\mathrm{M} \Omega$ ) as described elsewhere (Chang et al., 2014). Action potentials of single HL-1 cells were recorded in the current-clamp mode at a rate of $1 \mathrm{~Hz}$. Membrane currents and potentials were recorded using an integrating patch clamp amplifier (Axopatch 200B; Molecular Devices). Command pulses were generated by a 16-bit Digidata 1320A D/A converter (Molecular Devices) controlled by pCLAMP8.0.2 software (Molecular Devices).

Data acquisition and analysis were performed using the Clampex and Clampfit module of pCLAMP software, respectively. Recordings were filtered at $10 \mathrm{kHz}$ and acquired at $100 \mathrm{kHz}$. After forming the whole-cell recording configuration, a capacitive transient induced by a $10-\mathrm{mV}$ step from a holding voltage of $0 \mathrm{mV}$ was recorded and used for the calculation of cell capacitance. Series resistance was in the range of 4-6 M $\Omega$ and was compensated by $60 \%-80 \%$. Solution contents and pulse protocols for recording of specific ionic current are described in the Supplemental Material.

Chemicals. All chemicals used were purchased from Sigma-Aldrich (St. Louis, MO) except for fura-2-AM and Pluronic F127 which were purchased from Molecular Probes (Eugene, OR). Fura-2-AM and Pluronic F127 were dissolved in dimethylsulfoxide. Other drugs were dissolved in physiologic saline before the start of the experiment.

Statistical Analysis. Data are expressed as the mean \pm S.E.M. Statistical analysis was carried out by one-way ANOVA followed by post hoc Tukey's test for multiple comparisons. $P<0.05$ was considered statistically significant. Sigmaplot 12.0 (Systat Software, Chicago, IL) was used for fitting data with Boltzmann or other userdefined functions.

\section{Results}

General Features of Experimental Animals. During the 6th week after STZ injection, vehicle-treated diabetic rats had markedly lower body weight and higher fasting blood glucose levels compared with the age-matched nondiabetic controls. There was no significant change in body weight gain or blood glucose levels in either the diabetic or control group after AG administration for 5 weeks (Table 1). Diabetes was associated with lower LV weight (LVW) and heart weight $(\mathrm{HW})$ that were attributable to lower body weight (BW). AG-treated diabetic rats showed a significant decrease in LVW and HW, but did not differ significantly in BW from the untreated diabetic group. The atrial weight/BW, LVW/BW, and $\mathrm{HW} / \mathrm{BW}$ ratios in the diabetic rats were significantly 
A
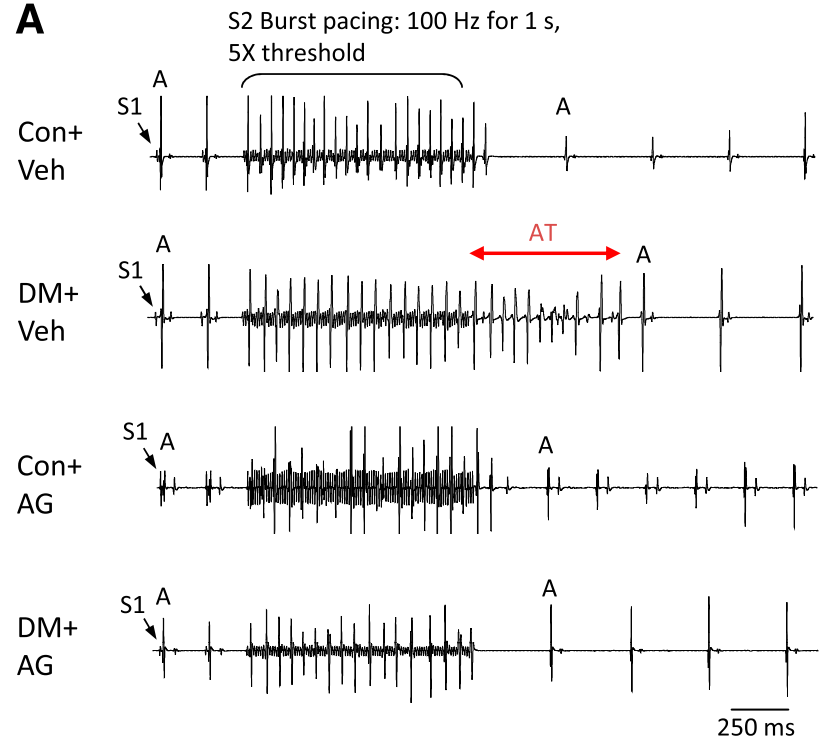

B

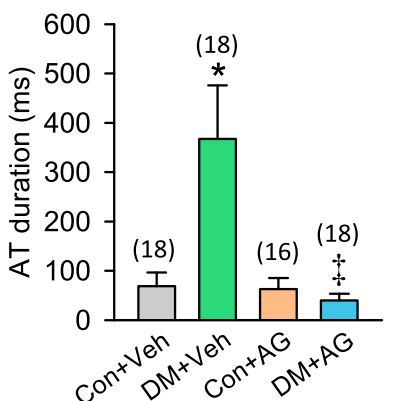

C

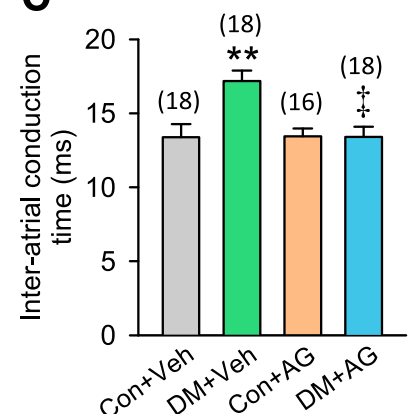

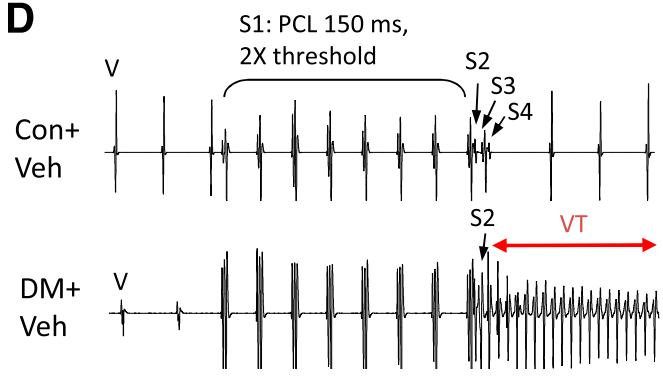
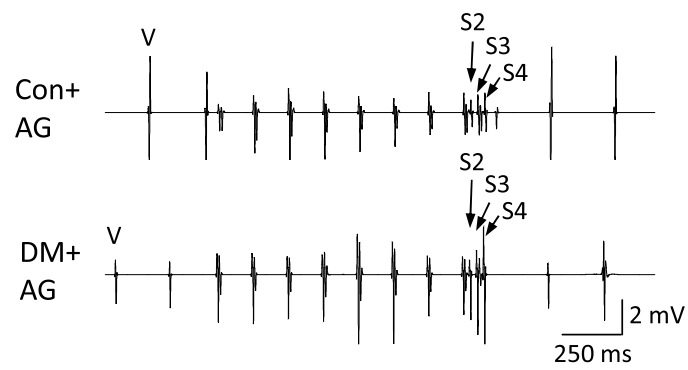

E

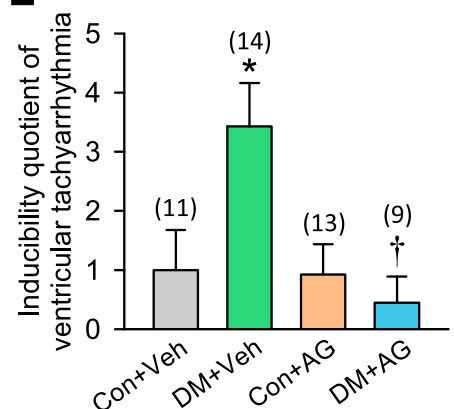

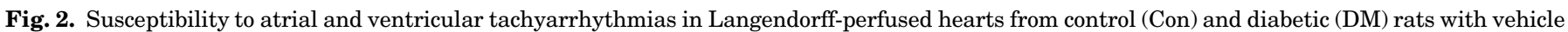

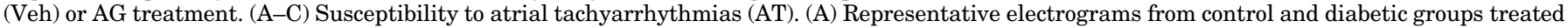

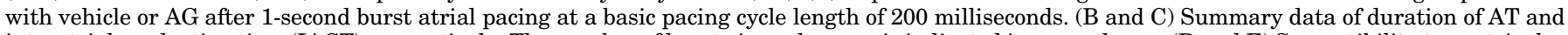

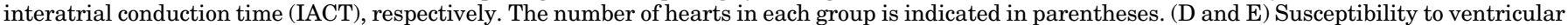

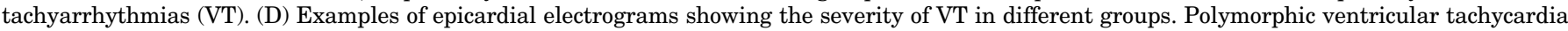

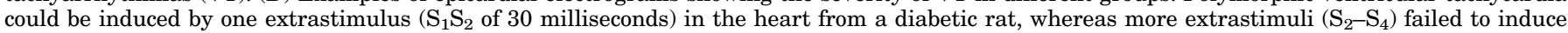

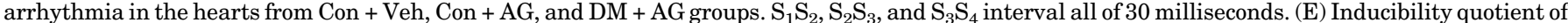

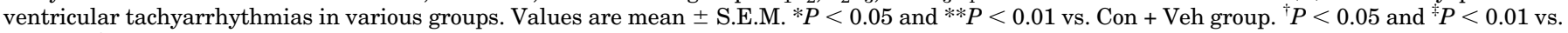
$\mathrm{DM}+$ Veh group.

higher compared with those of nondiabetic controls, and were reduced by treatment with AG (Table 1).

Representative left atrial and ventricular tissue sections from each group stained with Masson's trichrome are shown in Supplemental Fig. 1A. The amount of fibrosis in both tissues, as a percentage of the perimuscular interstitial blue area (except muscle for each rat heart), was significantly higher in the vehicle-treated diabetic group than in the control group (Supplemental Fig. 1, B and C). There was a significant reduction in cardiac fibrosis in AG-treated rats compared with their vehicle-treated diabetic counterparts.

Effects of AG on Hemodynamic and Electrocardiographic Indices. Diabetic rats had significantly decreased heart rate, LV end-systolic pressure and LV developed pressure, and maximal rate of rise $\left(\mathrm{d} P / \mathrm{d} t_{\max }\right)$ and fall $\left(\mathrm{d} P / \mathrm{d} t_{\min }\right)$ of $\mathrm{LV}$ pressure compared with nondiabetic controls. Diabetic rats also showed increased LV end-diastolic pressure and relaxation time constant (Tau) of LV. AG-treated diabetic rats exhibited improved LV end-systolic pressure, LV developed pressure, and relaxation Tau (Fig. 1A; Table 1), and also showed a trend toward higher $\mathrm{LV} \mathrm{d} P / \mathrm{d} t_{\max }$ and $\mathrm{d} P / \mathrm{d} t_{\min }$, and lower $\mathrm{LV}$ end-diastolic pressure, but the heart rates were not affected. AG administration significantly attenuated the prolongation of the QTc interval $(P<0.001)$ in diabetic rats (Fig. 1A; Table 1). Other parameters were similar in all groups. AG treatment had no significant effect on any measured parameters in nondiabetic controls.

Effects of AG on the Intracardiac Conduction System. Langendorff-perfused diabetic hearts had a longer basic cycle length, QT intervals, atrial effective refractory periods (AERP), AV nodal effective refractory period, His-Purkinje system functional refractory period, and ventricular effective refractory period (VERP) compared with nondiabetic controls (Fig. 1B; Table 2). No changes in other parameters were observed in any group. Administration of AG significantly prevented the prolongation of the QT interval, AERP, His-Purkinje system functional refractory period, and VERP in diabetic hearts. However, AG-treated diabetic rats showed a nonsignificant trend toward a shorter basic cycle length and AV nodal effective refractory period.

Effects of AG on Susceptibility to Atrial and Ventricular Tachyarrhythmias. Only a small number of atrial 

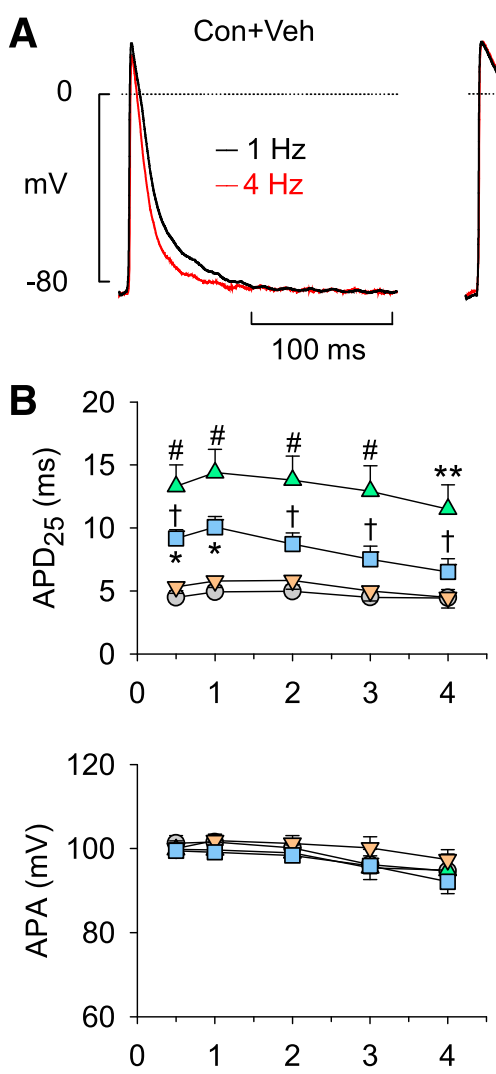

$\mathrm{DM}+\mathrm{Veh}$
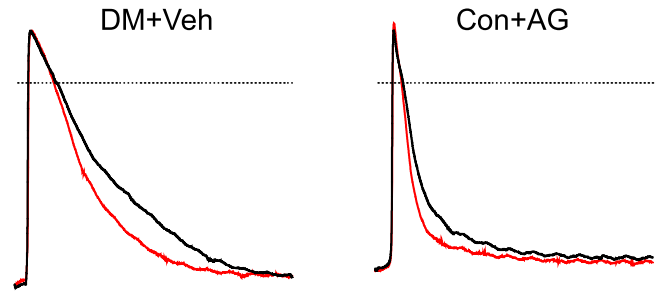

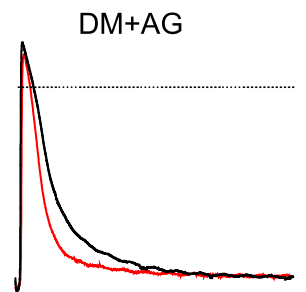

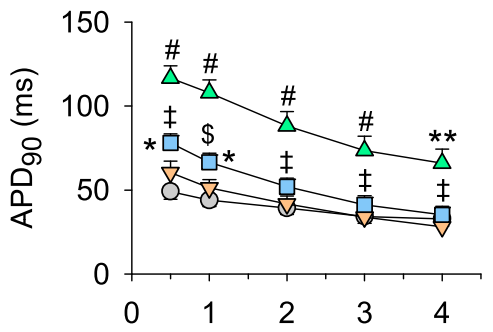
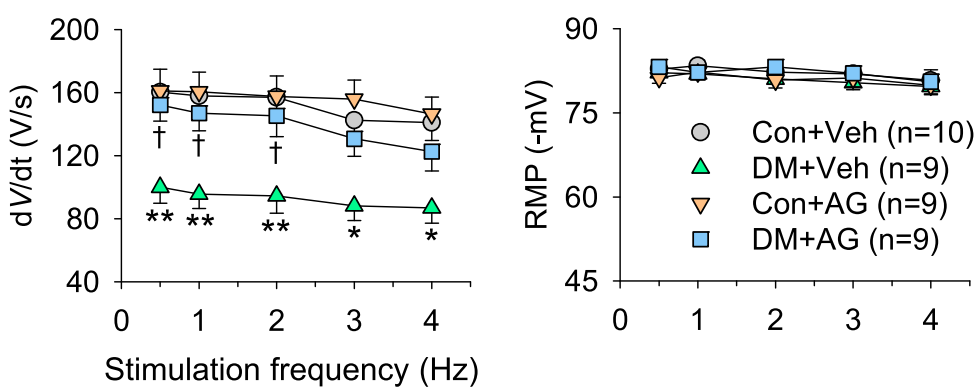

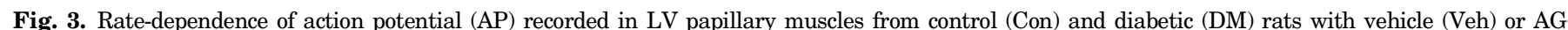

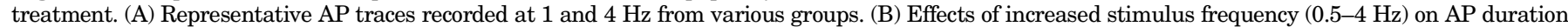

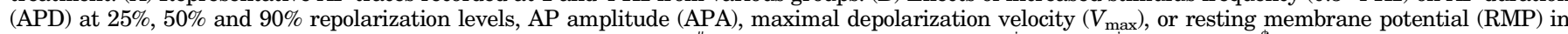

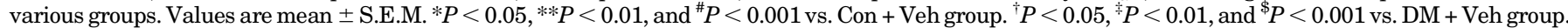

tachyarrhythmias (AT) were induced in control hearts. In contrast, the same stimulation induced a much longer duration of AT in diabetic hearts, along with a wide range in the values of this parameter (Fig. 2, A and B). The interatrial conduction time (IACT) was significantly longer in diabetic hearts than in controls (Fig. 2C). AG administration significantly reduced the duration of AT with normalization to IACT in diabetic hearts but did not affect the AT vulnerability and IACT in the control group.

Representative recordings of the induction of ventricular tachyarrhythmias are shown in Fig. 2D. Control rats with or without AG treatment had very low arrhythmia scores (Fig. 2, $\mathrm{D}$ and $\mathrm{E})$. In contrast, the inducibility quotient was significantly higher in diabetic hearts versus controls and was decreased by AG treatment.

Effects of AG on Action Potential Characteristics in Ventricular and Atrial Tissues. Representative recordings of ventricular action potentials during stimulation at 1 and $4 \mathrm{~Hz}$ are shown in Fig. 3A. The diabetic group had significantly prolonged action potential durations at the $25 \%, 50 \%$, and $90 \%$ repolarization levels $\left(\mathrm{APD}_{25}, \mathrm{APD}_{50}\right.$, and $\left.\mathrm{APD}_{90}\right)$ at all pacing frequencies tested $(0.5-4 \mathrm{~Hz})$ compared with those of the control group (Fig. 3). The maximal depolarization velocity $\left(V_{\max }\right)$ was lower in diabetic muscles compared with that of controls at each frequency (Fig. 3B). Administration of AG attenuated the prolongation of APD and decrease in $V_{\max }$ in diabetic rats but had no effect in the control group. Other parameters were not affected by either diabetes or AG treatment. Similar but smaller changes in APD and $V_{\max }$ were also observed in atrial strips from diabetic rats (Supplemental Fig. 2). AG attenuated the prolongation of $\mathrm{APD}_{50}$ and $\mathrm{APD}_{90}$ and depression of $V_{\text {max }}$, and also tended to attenuate the prolongation of $\mathrm{APD}_{25}$ in the diabetic group.

Effects of AG on Cell Shortening and Relengthening, and $\mathrm{Ca}^{2+}$ Transients in Cardiomyocytes. Diastolic cell lengths were smaller in LV myocytes from the diabetic group treated with vehicle or AG. Diabetic myocytes had significantly prolonged time-to-peak shortening and time-to-50\% relengthening, and showed a trend toward smaller fractional shortening compared with those of controls (Fig. 4, A and C). Fura-2 fluorescence data showed that diabetic myocytes displayed higher diastolic $\mathrm{Ca}^{2+}$ transient levels associated with longer times to peak and decay times of the $\mathrm{Ca}^{2+}$ transient than did controls (Fig. 4, A and B). In parallel with the fractional shortening, diabetic myocytes also showed a trend toward lower $\mathrm{Ca}^{2+}$ transient amplitude. AG administration significantly reversed the abnormal kinetic parameters of cell shortening/ relengthening and $\mathrm{Ca}^{2+}$ transients in diabetic myocytes. None of these parameters changed in the AG-treated control myocytes. Similar results on the basic properties of $\mathrm{Ca}^{2+}$ transients and cell contraction were observed in atrial myocytes from various groups (Supplemental Fig. 3). However, the amplitude of $\mathrm{Ca}^{2+}$ transients was significantly lower in the diabetic group compared with the controls. 
A Con+Veh $\mathrm{DM}+\mathrm{Veh}$ Con+AG $D M+A G$
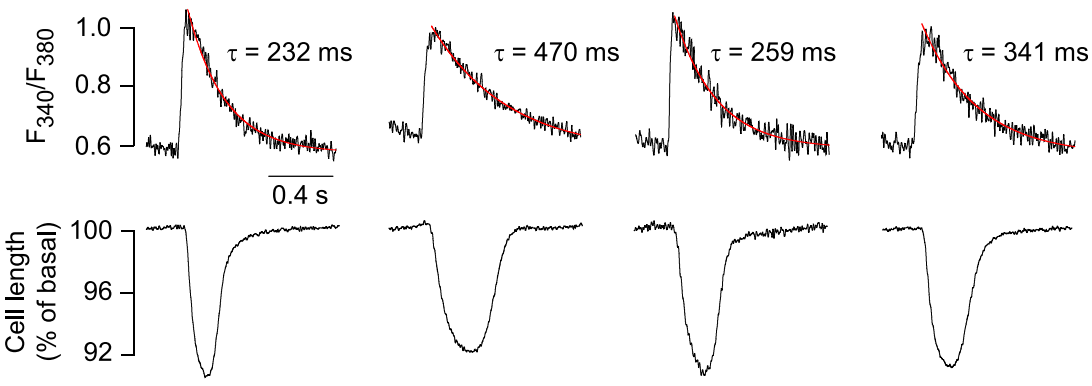

\section{B Diastolic $\mathrm{Ca}^{2+}$ transient}
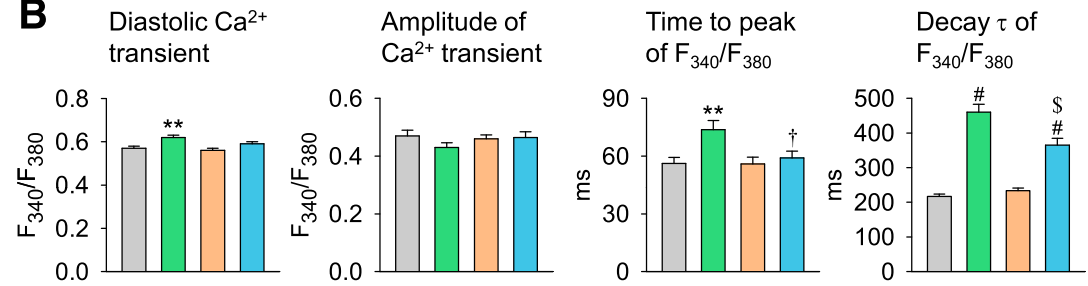

Fig. 4. Intracellular $\mathrm{Ca}^{2+}$ transients and contractile properties of LV myocytes isolated from hearts of various groups. (A) Representative $\mathrm{Ca}^{2+}$ transients (fura-2 fluorescence ratio $\mathrm{F}_{340} / \mathrm{F}_{380}$, upper panel) with fitted curves and cell-shortening (lower panel) traces recorded during field stimulation at $1 \mathrm{~Hz}$. (B and C) Intracellular $\mathrm{Ca}^{2+}$ transient (B) and contraction (C) properties of myocytes isolated from control [Con] + vehicle [Veh] $(n=40$ myocytes $/ 5$ rats), diabetes mellitus $[D M]+\operatorname{Veh}(n=52 / 8)$, Con + AG $(n=53 / 7)$, and DM + AG $(n=39 / 7)$ groups. Values are mean \pm S.E.M. ${ }^{*} P<0.05,{ }^{*} P<0.01$, and ${ }^{\#} P<0.001$ vs. Con + Veh group. ${ }^{\dagger} P<0.05,{ }^{\ddagger} P<0.01$, and ${ }^{\$} P<0.001$ vs. DM + Veh group.

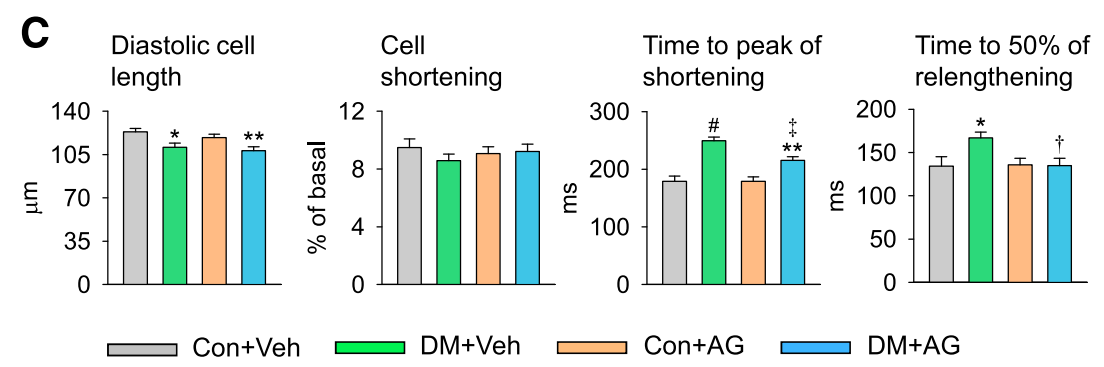

To assess the sarcoplasmic reticulum (SR) $\mathrm{Ca}^{2+}$ content, we rapidly applied caffeine $(10 \mathrm{mM}) 10$ seconds after cessation of electrical stimulation (Fig. 5). LV myocytes from diabetic rats had a tendency toward a smaller caffeine-induced $\mathrm{Ca}^{2+}$ transient amplitude, together with a markedly longer decay time constant compared with those of the controls. AG treatment partially attenuated the prolonged decay time in diabetic myocytes.

Effects of AG on the Expression of $\mathrm{Ca}^{2+}$ Handling Proteins. There was a significant decrease in the expression of SERCA2a protein in LV tissues from diabetic rats compared with the nondiabetic control group, whereas PLB (total and phosphorylated), RyR2, and NCX were unchanged (Fig. 6, A-E). AG administration significantly restored the expression of SERCA2a but did not affect the levels of PLB, pSer16-PLB, RyR2, or NCX in diabetic tissues. AG treatment did not affect the expression of any protein tested in the control group.

Effects of AG on AGEs Accumulation and Superoxide Production in LV Tissues. There was a significant increase in the level of AGEs in LV tissues of diabetic rats, and this was attenuated after AG treatment (Fig. 6F). AG had no effect on AGE levels in control rats. The NADPH-dependent superoxide production in LV tissues (10 rats per group) was evaluated using a lucigenin-enhanced chemiluminescence technique and expressed as values relative to control group. Diabetes induced a $1.75 \pm 0.09$-fold increase in superoxide generation compared with controls $(P<0.001)$. AG treatment attenuated diabetes-induced superoxide production $(1.29 \pm$ 0.14 -fold, $P<0.05$ vs. diabetes + vehicle group), but it did not affect the level in control rats $(1.02 \pm 0.09$-fold $)$.
Effects of AG on High-Glucose-Induced AGEs Formation and Oxidative Stress in HL-1 Atrial Cells. To further explore the effect of AG on diabetes-induced AGEs and ROS production in vitro, we treated HL-1 cells with high glucose (HG, $25 \mathrm{mM}$ ) to model the diabetic condition. Mannitol $(19.4 \mathrm{mM})$ with normal glucose $(\mathrm{NG}, 5.6 \mathrm{mM})$ was used in parallel to test whether the effect of HG was simply due to changes in medium osmolality. Western blotting and fluorescence study showed that HG incubation enhanced the AGEs and ROS production, and this increase was significantly attenuated by AG $(1 \mu \mathrm{M})$ treatment (Fig. 7, A and B). The increase of AGEs and ROS production was not related to increased osmolality because mannitol did not alter this effect.

To explore the role of RAGE in AGEs-mediated effects, cells were transfected with siRNA-RAGE for 48 hours to silence RAGE, and then incubated with HG for 24 hours. Knockdown of RAGE was confirmed by a decrease in RAGE protein level. AG administration had no effect on RAGE expression either in control or RAGE gene knockdown cells (Fig. 7C). Knockdown of the RAGE gene significantly attenuated HG-induced production of ROS (Fig. 7D). AG treatment in control cells induced a slightly higher decrease in HG-induced ROS production compared with cells with RAGE gene knockdown. Moreover, AG administration further decreased ROS production in RAGE gene knockdown cells (Fig. 7D), which could be due to the less AGEs to interact with the residual RAGE.

Effects of AG on High-Glucose-Induced APD Prolongation in HL-1 Atrial Cells. To evaluate the effect of AG on the changes in APD under HG conditions and whether this 

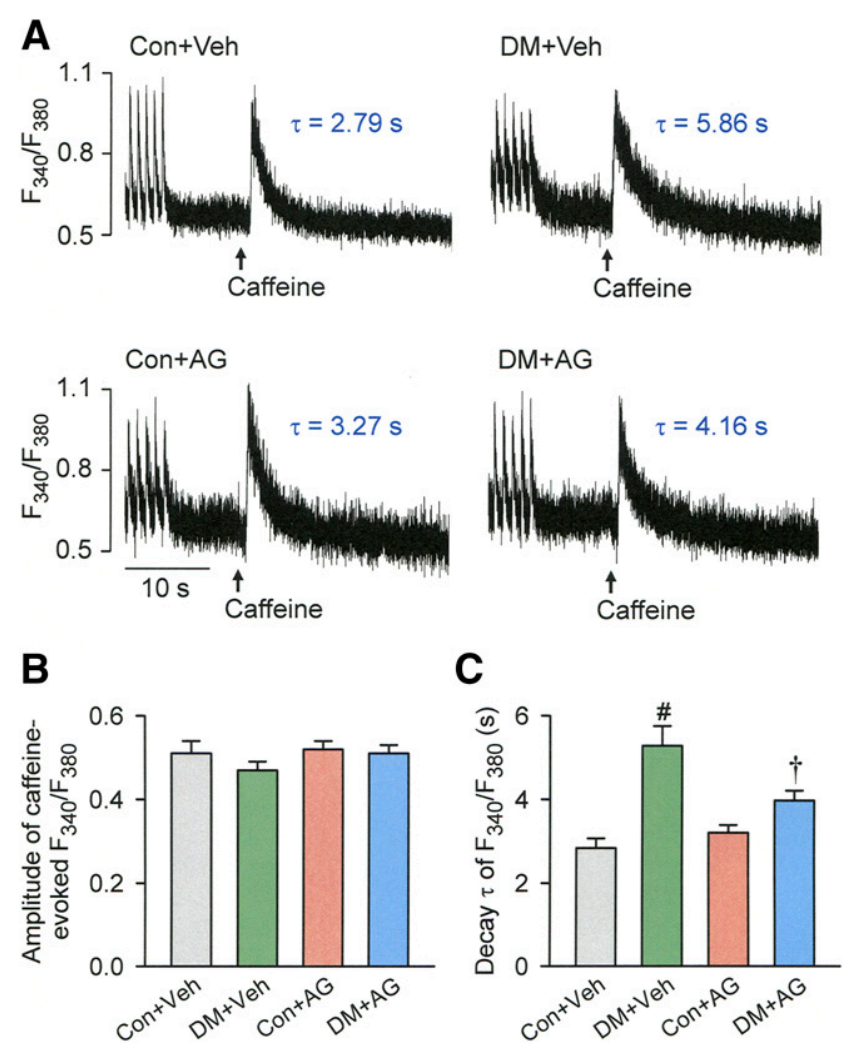

Fig. 5. Caffeine-induced intracellular $\mathrm{Ca}^{2+}$ transients of $\mathrm{LV}$ myocytes isolated from hearts of various groups. (A) Representative tracings of caffeine-induced $\mathrm{Ca}^{2+}$ transients. Myocytes were first stimulated at $1 \mathrm{~Hz}$ and caffeine $(10 \mathrm{mM})$ was rapidly applied 10 seconds after cessation of electrical stimulation. (B and $\mathrm{C}$ ) Bar graphs showing the averaged data of the amplitude and decay time constant $(\tau)$ of the caffeine-induced $\mathrm{Ca}^{2+}$ transients in myocytes from control [Con] + vehicle [Veh] $(n=24$ myocyte $/ 5$ rats $)$, diabetes mellitus $[\mathrm{DM}]+\operatorname{Veh}(n=29 / 7)$, Con + AG $(n=33 / 7)$, and $\mathrm{DM}+\mathrm{AG}(n=23 / 6)$ groups. Values are mean \pm S.E.M. ${ }^{\#} P<0.001 \mathrm{vs}$. Con + Veh group. ${ }^{\dagger} P<0.05$ vs. $\mathrm{DM}+$ Veh group.

effect was related to the AGE-RAGE pathway, the action potentials were recorded in HL-1 cells incubated with NG or HG and in cells cultured in HG with or without RAGE gene knockdown as described above. The electrophysiologic features of murine HL-1 cells resemble those of primary cardiomyocytes, with many typical ionic currents including $I_{\mathrm{Na}}, I_{\text {to }}$, $I_{\mathrm{Ca}}$, and the rapidly activating $I_{\mathrm{K}}\left(I_{\mathrm{Kr}}\right)$ (Yang et al., 2005). The APD was longer in cells cultured in HG compared in cells cultured in NG (Fig. 8, A and B). The prolongation of APD was partially attenuated by AG (1 $\mu \mathrm{M})$ treatment (Fig. 8, A and B). Incubation with mannitol had no effect on APD. Knockdown of the RAGE gene alone attenuated the prolongation of APD in cells cultured in HG (Fig. 8, C and D). AG treatment in control (Con si) cells attenuated HG-induced prolongation of APD. AG administration further tended to attenuate the prolongation of APD in RAGE gene knockdown cells cultured in HG (Fig. 8D).

Effects of AG on Whole-Cell $\mathrm{K}^{+}$Currents in Cardiomyocytes. The current densities of both $I_{\text {to }}$ and $I_{\mathrm{SS}}$ were considerably smaller in LV myocytes isolated from diabetic hearts compared with those from controls at each voltage. However, the inward rectifier $\mathrm{K}^{+}$current $\left(I_{\mathrm{K} 1}\right)$ density was not significantly changed in the diabetic group (Fig. 9, A-C). AG treatment of the diabetic rats significantly restored densities of both $I_{\text {to }}$ and $I_{\mathrm{SS}}$. Neither $I_{\text {to }}$ nor $I_{\mathrm{SS}}$ density was altered after
AG administration in the control group. Similar results in $I-V$ relationships were observed in atrial myocytes isolated from animals in various groups (Supplemental Fig. 4). The rate of decay of $I_{\text {to }}$ at each voltage in LV myocytes was not significantly affected either by diabetes or by AG treatment (Fig. 9D).

The normalized conductance-voltage curves were calculated from the $I_{\text {to }}$ amplitude in Fig. 9B. The estimated maximal $I_{\text {to }}$ channel conductance $\left(G_{\text {to, max }}\right)$ was significantly smaller in diabetic LV myocytes than in controls, and was partially restored by AG treatment (Supplemental Table 1). Neither diabetes nor AG treatment modified the steady-state activation curve, or made a significant difference in the half-activation potential $\left(V_{\mathrm{h}}\right)$ or slope factor $(k)$ between the groups (Fig. 9E; Supplemental Table 1). The voltage-dependence of steady-state inactivation of $I_{\text {to }}$ was measured by a double-pulse protocol (Fig. 9F inset). Similarly, neither diabetes nor AG treatment significantly altered the steady-state inactivation curve, and there was no difference in the half-inactivation $V_{\mathrm{h}}$ or $k$ value between the groups (Fig. 9F; Supplemental Table 1).

To test the possibility that the decrease in $I_{\text {to }}$ density is a change in the time-dependence of recovery from inactivation, we studied the recovery properties of $I_{\text {to }}$ using a paired-pulse protocol (Fig. 9G inset). The results are shown in Fig. 9G, where the fraction of recovery current amplitude is reported as a function of recovery time. Fitting data with a monoexponential function of these curves enables derivation of similar recovery time constants in all groups (Supplemental Table 1).

Effects of AG on L-Type $\mathrm{Ca}^{2+}$ Current $\left(I_{\mathrm{Ca}, \mathrm{L}}\right)$ in $\mathbf{L V}$ Myocytes. Families of representative $I_{\mathrm{Ca}, \mathrm{L}}$ current traces from various groups are shown in Supplemental Fig. 5A. The peak current density of $I_{\mathrm{Ca}, \mathrm{L}}$ at different membrane potentials is quantified in an $I-V$ relation plot (Supplemental Fig. 5B). There were no significant differences in $I-V$ relation between the control and diabetic groups at all voltages. There was also no significant difference in the time course of the fast and slow components of $I_{\mathrm{Ca}, \mathrm{L}}$ inactivation. AG administration did not affect the $I-V$ curve and inactivation time courses in either the control or diabetic groups. Similarly, there were no differences in the voltage-dependency of the steady-state activation and inactivation or in the recovery from inactivation kinetics (Supplemental Fig. 5, C and D) of $I_{\mathrm{Ca}, \mathrm{L}}$ among each group. All the mean data are presented in Supplemental Table 1.

\section{Discussion}

The major finding of this study is that treatment with AG, a prototype inhibitor of AGEs formation, markedly protected against the electromechanical remodeling that leads to cardiac dysfunction and arrhythmogenesis in diabetic rats. AG attenuated cardiac hypertrophy and fibrosis, improved both diastolic and systolic function, attenuated abnormal $\mathrm{Ca}^{2+}$ handling and cell contraction kinetics, normalized the changes in the repolarization phase of action potentials, and restored $\mathrm{K}^{+}$currents in diabetic hearts by primarily inhibiting AGE and ROS formation.

Both diastolic and systolic dysfunction have been described in patients and experimental animals with type 1 and type 2 diabetes (Bugger and Abel, 2014; Huynh et al., 2014). The present in vivo study demonstrated that diabetes was associated with impaired ventricular relaxation and depressed systolic performance. AG administration offered significant 
A
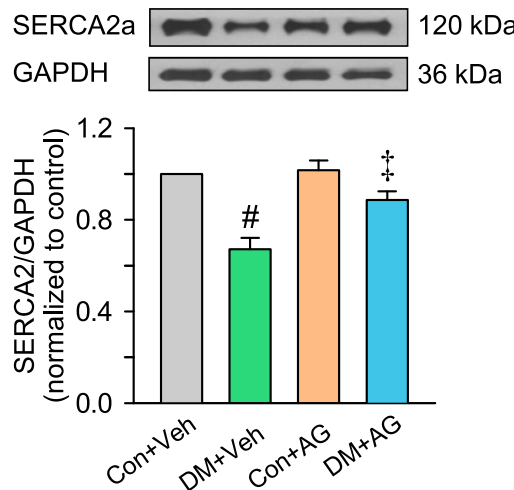

D
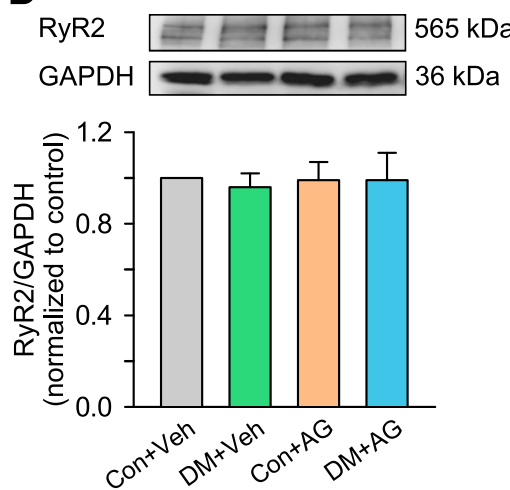

B
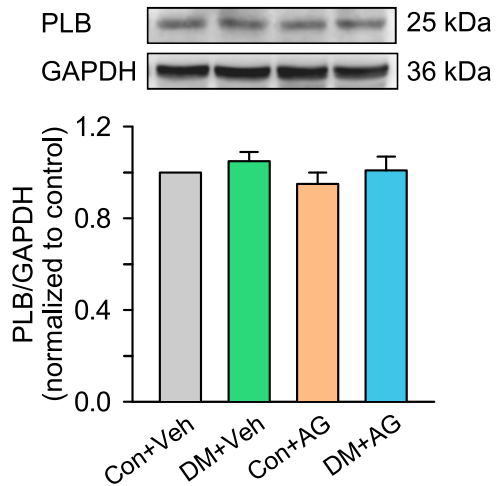

E

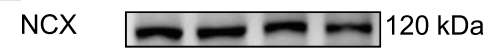

C

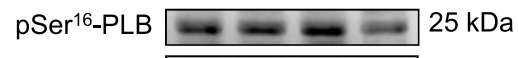

GAPDH $20 \mathrm{kDa}$

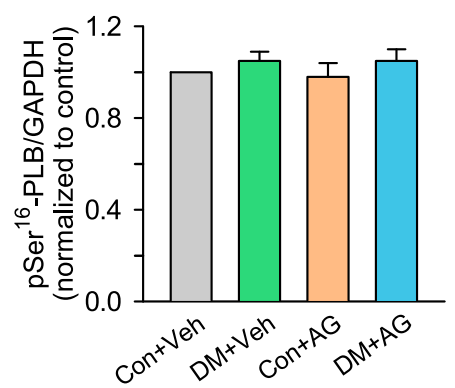

$\mathbf{F}$
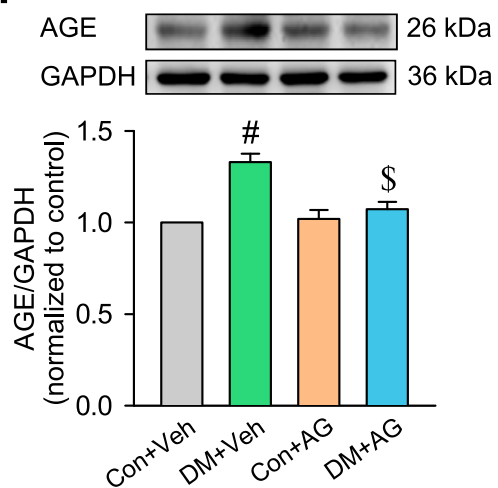

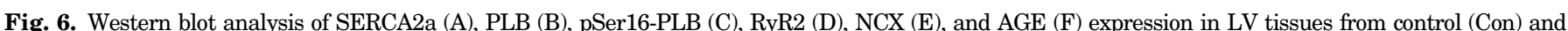

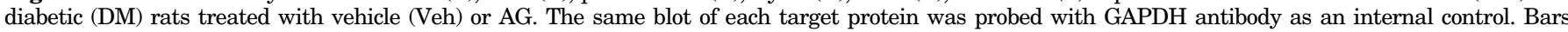

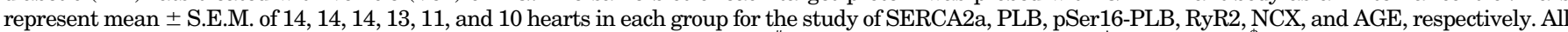

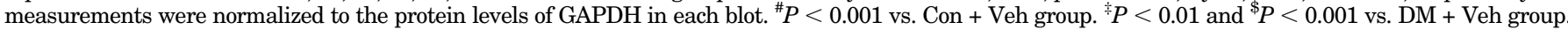

hemodynamic benefits although it only mildly improved LV contractility $\left(\mathrm{d} P / \mathrm{d} t_{\max }\right)$. We also observed that the most significant changes in contraction and the $\mathrm{Ca}^{2+}$ transient in diabetic cardiomyocytes were the increases in rising and decay time, which characterize the most consistent hallmark of diabetic cardiomyopathy (Choi et al., 2002; Yaras et al., 2005; Shao et al., 2007; Zhang et al., 2008). The initial global diastolic dysfunction could be mainly related to impaired relaxation of myocytes, although mild LV fibrosis could also contribute. Intervention with AG could ameliorate these diastolic changes. Because the amplitudes of $\mathrm{Ca}^{2+}$ transients, SR $\mathrm{Ca}^{2+}$ content, and shortening of diabetic LV myocytes were not significantly impaired as reported previously elsewhere (Ishikawa et al., 1999; Zhang et al., 2008), the systolic dysfunction may not be simply attributable to defects of the myocytes. It is likely that myocyte apoptosis (Sorrentino et al., 2017), which could be attenuated by AG treatment (Shi et al., 2013), as well as other noncardiomyocyte factors may play a predominant role in the present stage of diabetes.

During the excitation-contraction coupling process, SR SERCA2a facilitates relaxation and thereby makes $\mathrm{Ca}^{2+}$ available for the next wave of contraction (Bers, 2002). Phosphorylation of the SERCA2a inhibitor PLB relieves the inhibitory effect exerted by $\mathrm{PLB}$, which enhances $\mathrm{Ca}^{2+}$ uptake. The unchanged $I_{\mathrm{Ca}, \mathrm{L}}$ observed in diabetic myocytes suggests that alterations in $\mathrm{Ca}^{2+}$ handling are mediated by altered $\mathrm{SR}$ release/uptake mechanisms. The increase in rising time of both the $\mathrm{Ca}^{2+}$ transient and contraction in diabetic myocytes may be partly due to the altered function and/or expression of RyR2 on SR (Choi et al., 2002; Yaras et al., 2005). Our data showed no major changes in RyR2 expression in diabetic hearts, suggesting that the defect in RyR2 function, which may have been caused by its cross-linking with AGEs (Bidasee et al., 2003), may be a contributory factor. Therefore, AG-mediated restoration of RyR2 function is likely caused via inhibition of AGEs formation.

The diabetic heart is characterized by compromised function and expression of SERCA2a (Zhong et al., 2001; Choi et al., 2002; Bidasee et al., 2004). Our current finding of an overt reduction in SERCA2a protein despite unchanged levels of PLB could explain the diabetes-induced delayed $\mathrm{Ca}^{2+}$ clearing and relaxation. AG effectively normalized these altered kinetics partially mediated by the restoration of SERCA2a levels or by attenuating AGEs formation and their subsequent cross-linking on SERCA2a (Bidasee et al., 2004) or AGE-RAGE mediated activity (Petrova et al., 2002). We also found that the decay rate of the caffeine-induced $\mathrm{Ca}^{2+}$ transient, which reflects the ability of $\mathrm{Ca}^{2+}$ extrusion of NCX (Sorrentino et al., 2017), was slower in diabetic myocytes, implying that despite normal expression NCX activity may be impaired, and this could be partially restored with AG treatment. Overall, AG administration should be expected to prevent the disturbance of $\mathrm{Ca}^{2+}$ homeostasis through the restoration of function or expression of $\mathrm{Ca}^{2+}$ handling proteins. 
A
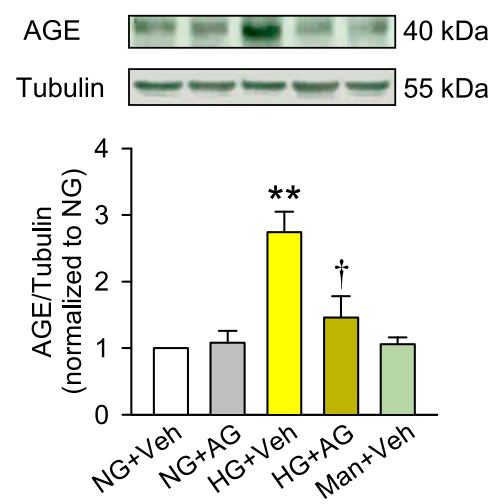

C
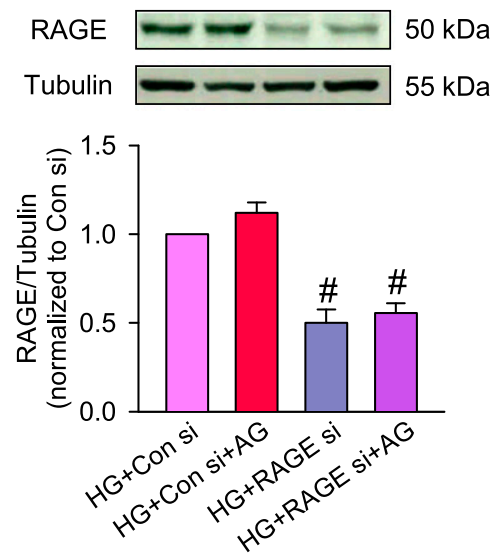

B

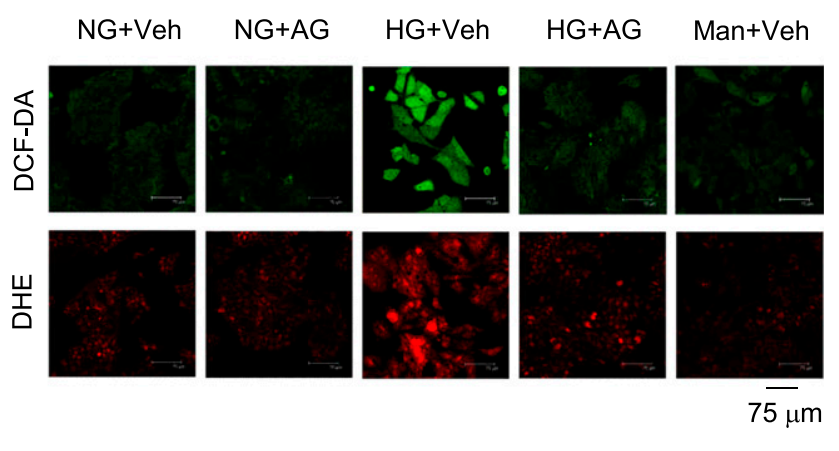

D

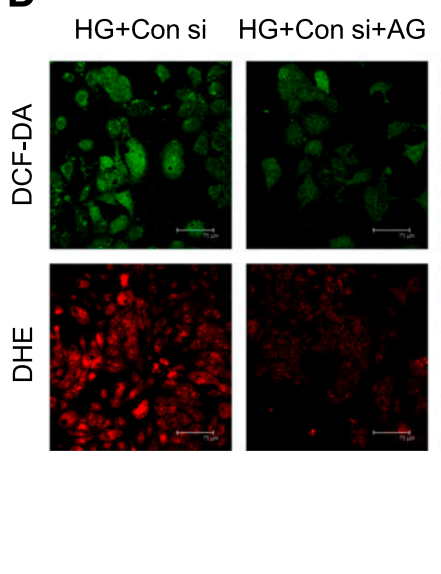

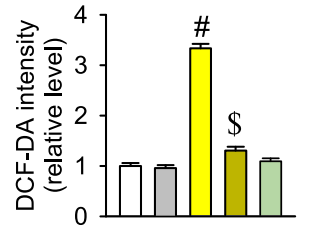
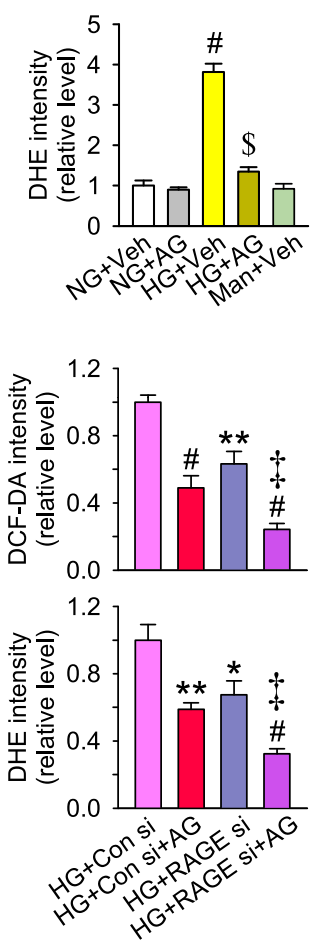

Fig. 7. Effects of AG on HG-induced AGE formation and ROS production (A and B) and effects of RAGE knockdown on HG-induced ROS production (C and D) in HL-1 cells. (A and B) (A) Western blot analysis of AGE expression in myocytes incubated with NG (5.6 mM), HG (25 mM), or mannitol (Man; $19.4 \mathrm{mM}$ mannitol with $5.6 \mathrm{mM}$ glucose, the osmotic control) for 24 hours and subsequent treatment with vehicle or $1 \mu \mathrm{M}$ AG for the last 12 hours. The same blot of the target protein was probed with tubulin antibody as an internal control. All measurements were normalized to the levels of tubulin in each blot. The relative expression level was normalized to that of $\mathrm{NG}+$ vehicle (Veh) group. Each value represents the mean \pm S.E.M. of five independent experiments. (B) Intracellular ROS production was measured by 2,7-dichlorodihydrofluorescein diacetate (DCF-DA, cytoplasm staining) or dihydroethidium (DHE, nucleus staining) fluorescent dye using confocal microscope (left panel) in cells treated with the identical conditions as in $A$. Relative fluorescence density was quantified (right panel). Each value represents the mean \pm S.E.M. of five independent experiments. $* * P<0.01$ and ${ }^{\#} P<0.001$ vs. NG + Veh group. ${ }^{\dagger} P<0.05$ and ${ }^{\$} P<0.001$ vs. HG + Veh group. (C and D) (C) After transfected with indicated siRNA for 48 hours, cells were incubated with HG for 24 hours and subsequent treatment with vehicle or $1 \mu \mathrm{M}$ AG for the last 12 hours, the expression of RAGE or tubulin was evaluated by Western blotting (upper panel). The relative expression level was normalized to the level of control siRNA (Con si) group. Bars represent mean \pm S.E.M. of five independent experiments in each group. (D) Intracellular ROS production was measured by 2,7-dichlorodihydrofluorescein diacetate (DCF-DA) or dihydroethidium (DHE) (left panel) in cells with conditions as in (C). Relative fluorescence density was quantified (right panel). Each value represents the mean \pm S.E.M. of five independent experiments. ${ }^{*} P<0.05,{ }^{* *} P<0.01$, and ${ }^{\sharp} P<0.001$ vs. HG + Con si group. ${ }^{\sharp} P<0.01$ vs. HG + RAGE si group.

The important role of AGE-RAGE axis in diabetic cardiomyopathy has been revealed in RAGE gene-knockdown diabetic mice (Ma et al., 2009). The interaction of AGEs and RAGE may activate a diverse array of signals associated with increased oxidative stress and inflammation, and impaired $\mathrm{Ca}^{2+}$ homeostasis (Brownlee, 2001; Bodiga et al., 2014). In cultured HL-1 cells, we demonstrated that HG enhanced formation of AGEs as well as ROS production, which is in line with the findings from in vivo diabetes. Increased ROS production could be attenuated by knockdown of the RAGE gene or by AG treatment, suggesting that interaction of AGEs with RAGE could trigger this response. A similar mechanism could explain why the restoration of prolonged APD by AG was mimicked by knockdown of the RAGE gene in HG-cultured cell model. On the other hand, RAGE activation has been shown to enhance nitric oxide production and cell damage by up-regulation of inducible nitric oxide synthase (Sumi and Ignarro, 2004; Hegab et al., 2017). However, a previous study showed no increase in nitric oxide production in STZ-induced diabetic rat hearts (Stadler et al., 2005), implicating that the protective effect of AG in the diabetic heart may not necessarily due to inducible nitric oxide synthase inhibition.

Our results showed a substantial prolongation in APD in diabetic atrial and ventricular preparations as reported elsewhere (Pacher et al., 1999; Howarth et al., 2009). The longer QTc interval and VERP in diabetic rats were closely correlated with the longer ventricular APD. The lengthening of the APD in diabetes results mainly from a decrease in $I_{\text {to }}$ and, to a lesser extent, $I_{\mathrm{SS}}$ and/or delayed outward $\mathrm{K}^{+}$current $\left(I_{\mathrm{K}}\right)$ (Jourdon and Feuvray, 1993; Wang et al., 1995; Li et al., 2005). Our study confirmed a depression of $I_{\mathrm{to}}$ and $I_{\mathrm{SS}}$ densities in diabetic myocytes. The decrease in peak $I_{\text {to }}$ 
A

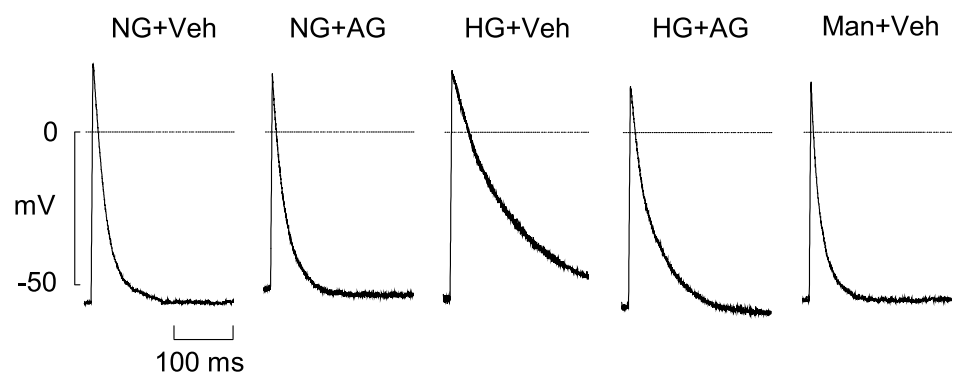

C

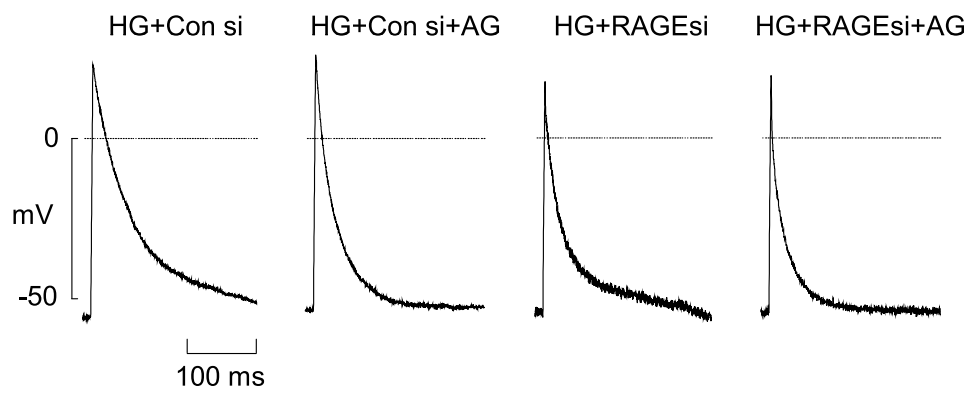

B
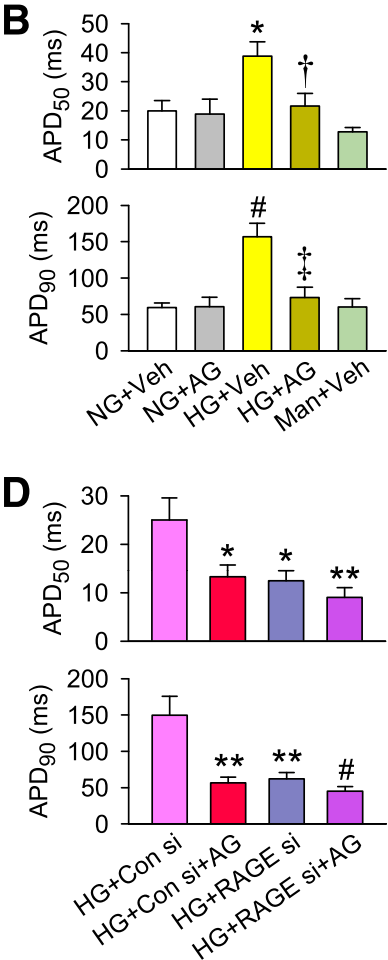

Fig. 8. Effects of AG on HG-induced AP remodeling (A and B) and effects of RAGE knockdown on HG-induced AP remodeling (C and D) in HL-1 cells. Cells were treated as the same conditions shown in Fig. 7. (A and B) (A) Representative AP traces recorded at $1 \mathrm{~Hz}$ from cells incubated with NG (5.6 mM glucose), HG (25 mM glucose), or mannitol (Man, $19.4 \mathrm{mM}$ mannitol with $5.6 \mathrm{mM}$ glucose) with vehicle (Veh) or AG (1 $\mu \mathrm{M})$ treatment. (B) Averaged AP duration (APD) at $50 \%$ and $90 \%$ repolarization levels in various groups. Bars represent mean \pm S.E.M. of eight cells in each group. $* P<0.05$ and ${ }^{\#} P<$ 0.001 vs. $\mathrm{NG}+$ Veh group. ${ }^{\dagger} P<0.05$ and ${ }^{\ddagger} P<0.01 \mathrm{vs}$. HG + Veh group. (C and D) (C) Representative AP traces recorded at $1 \mathrm{~Hz}$ in control (Con si) or RAGE knockdown (RAGE si) cells incubated with HG with or without AG $(1 \mu \mathrm{M})$ treatment. (D) Averaged APD at $50 \%$ and $90 \%$ repolarization levels in various groups. Bars represent mean \pm S.E.M. of eight cells in each group. ${ }^{*} P<0.05$, ${ }^{* *} P<0.01$, and ${ }^{\#} P<0.001$ vs. HG + Con si group.

density could result from either a lower density of $I_{\text {to }}$ channels (Qin et al., 2001; Howarth et al., 2009) or from modified properties of single channels-or both. Because we found no significant changes in the kinetics of activation, inactivation, or recovery of $I_{\text {to }}$, the former possibility seems to be more likely.

The most innovative finding of our study is that AG treatment markedly normalized the prolonged QT interval and APD in diabetic rats mainly through the restoration of $I_{\text {to }}$ and $I_{\mathrm{SS}}$ densities, possibly by attenuating ROS production. Our cultured cell study further reinforce the notion that the AGE-RAGE-ROS axis may involve in the HG-induced remodeling of APD, although other pathways such as glycosylation of $\mathrm{K}^{+}$channels should also be considered. We and others (Yaras et al., 2005; Shao et al., 2007) also found no significant differences in densities or any kinetic properties of $I_{\mathrm{Ca}, \mathrm{L}}$ between the control and diabetic groups, which excluded its putative role in the prolongation of APD. Additionally, AG improved the diminished $V_{\text {max }}$ in diabetic cardiac muscles, which could partially explain the restorations of myocardial refractoriness and interatrial conduction function.

Previous studies have shown that delayed atrial conduction and increased fibrotic deposition in the atria play a major role in promoting atrial tachyarrhythmias in diabetic rats (Kato et al., 2006). Recently, Watanabe et al. (2012) suggested that the increased spatial dispersion and alternans of atrial APD may also be key mechanisms. Our study showed that the interatrial conduction time and AERP were significantly longer and were combined with interstitial fibrosis in diabetic rat hearts.
Because moderate AERP prolongation seemed to be a factor against arrhythmogenesis, our results imply that the prominent conduction delay may be a major substrate for atrial tachyarrhythmias. Furthermore, the longer APD in diabetic atrial and ventricular preparations may produce early after-depolarizations (Lacombe et al., 2007), while the abnormal subcellular $\mathrm{Ca}^{2+}$ handling may predispose to delayed after-depolarizations, both of which may further promote arrhythmogenic triggered activities. Thus, we suggest that AG attenuates arrhythmogenic vulnerabilities, presumably via the prevention of abnormal structural remodeling, conduction velocity, AP duration, and $\mathrm{Ca}^{2+}$ handling.

Under hyperglycemic settings, the accelerated accumulation of AGEs in tissues plays an important role in the pathogenic process of diabetes and its complications. Moreover, the levels of plasma AGEs reflect the severity of the diabetic complications and independently predict mortality or hospitalization for diabetic patients compromised with heart failure (Willemsen et al., 2012). Thus, intervention with AG aimed at decreasing AGEs should confer clinical relevance and would be a novel approach to prevent the AGEs-mediated electrical and $\mathrm{Ca}^{2+}$ handling disorders in diabetic patients. By using a low to moderate dosage of AG (100 mg/kg per day), which was adopted from that used in experimental studies (range: $25-500 \mathrm{mg} / \mathrm{kg}$ per day), a beneficial antiremodeling effect was achieved in our diabetic rat model. Much lower doses ( $\sim 25$ - to 50-fold lower) recommended for use in humans should be expected, according to the consideration scale phenomenon (Mordenti, 1986). Effective and tolerated dosage 
A

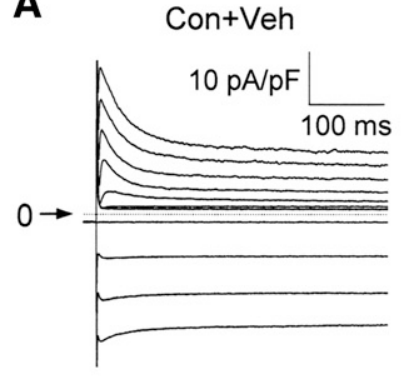

$\mathrm{DM}+\mathrm{Veh}$

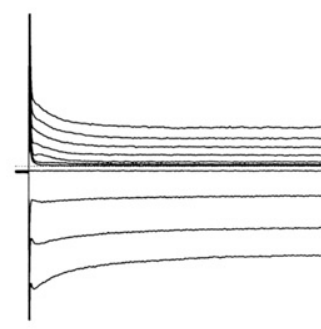

Con+AG

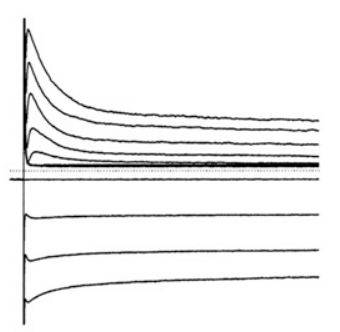

$\mathrm{DM}+\mathrm{AG}$

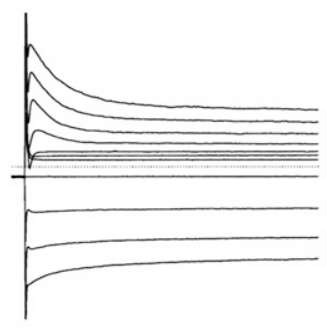

B

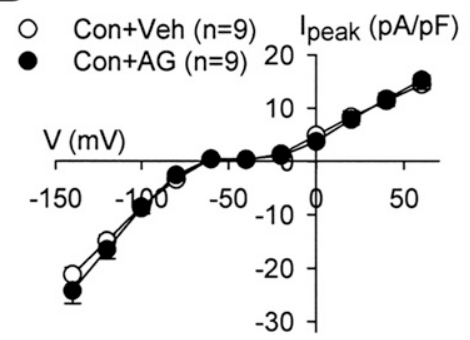

C

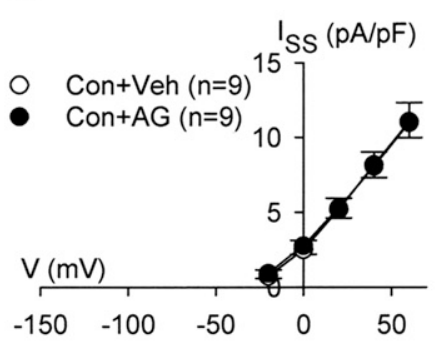

D
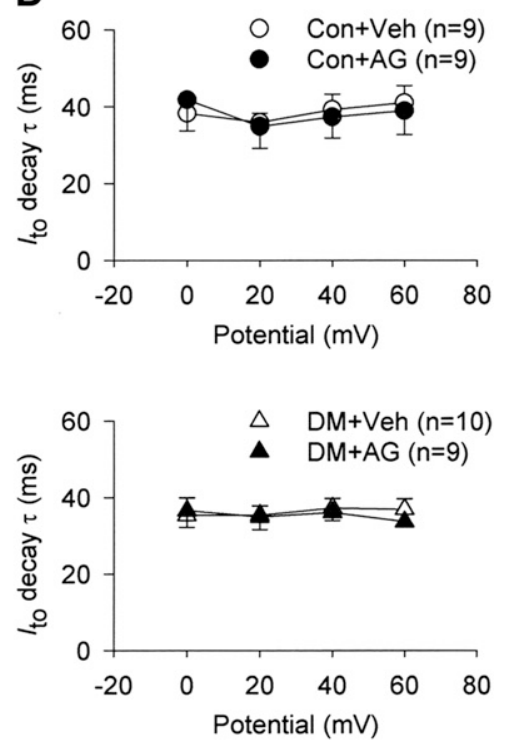

G

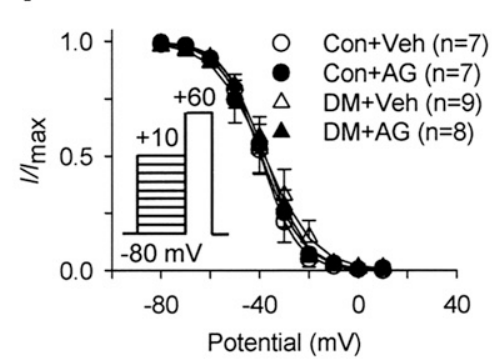

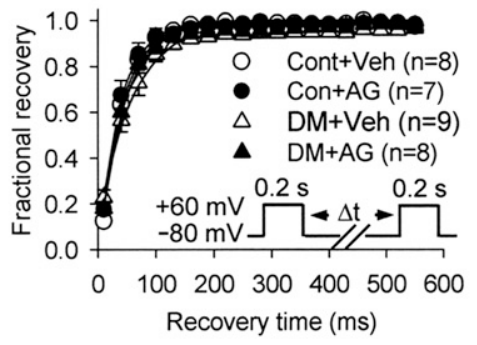

Fig. 9. Whole-cell $\mathrm{K}^{+}$currents recorded in $\mathrm{LV}$ myocytes from control (Con) and diabetic (DM) rats treated with vehicle (Veh) or aminoguanidine (AG). (A) Representative normalized $\mathrm{K}^{+}$current traces in myocytes from Con + Veh $(227 \mathrm{pF}), \mathrm{DM}+\mathrm{Veh}(223 \mathrm{pF})$, Con $+\mathrm{AG}(236 \mathrm{pF})$, and DM + AG $(165 \mathrm{pF})$ rats. (B) Average current density-voltage $(I-V)$ relationships of $I_{\mathrm{to}}$ and $I_{\mathrm{K} 1}$ peak currents. $I_{\mathrm{to}}$ was measured as the difference between the peak current and the steady-state current at the end of the pulse. (C) Average $I-V$ relationships of $I_{\mathrm{SS}}$ current. $I_{\mathrm{SS}}$ was measured as the steady-state current at the end of the pulse. (D) Voltage-dependent decay kinetics of $I_{\text {to }}$ from various groups. Data were obtained by fitting with a monoexponential function. (E and F) Steadystate activation (E) and inactivation (F) curves of $I_{\text {to }}$ in $L V$ myocytes from various groups. The activation curves were derived using $I-V$ curves shown in (B). Steady-state inactivation was examined with a double-pulse protocol (inset). The relative peak amplitudes of $I_{\mathrm{to}}$ were plotted against prepulse potentials. Smooth lines represent the fit of a Boltzmann function to the data. (G) Fractional recovery $\left(I_{\text {test pulse }} / I_{\text {prepulse }}\right)$ of $I_{\text {to }}$ from inactivation plotted as a function of recovery time in myocytes from various groups. Recovery of $I_{\text {to }}$ was determined with the paired-pulse protocol as shown in the inset. Smooth lines represent the fit of a monoexponential function to the data. Data are mean \pm S.E.M. ${ }^{* *} P<0.01$ and ${ }^{\#} P<0.001$ vs. Con + Veh group. ${ }^{\dagger} P<0.05$ vs. $\mathrm{DM}+$ Veh group.

levels for AG in management of patients with diabetic cardiomyopathy still need to be determined.

In summary, this study presented the first evidence that chronic administration of AG normalizes the electrical remodeling of the diabetic heart by restoring the diminished $\mathrm{K}^{+}$current density, and resolves mechanical dysfunctions by restoring the altered kinetics of $\mathrm{Ca}^{2+}$ handling and structural remodeling. AG-mediated attenuation of the atrial and ventricular arrhythmogenicities may be primarily attributed to its antiremodeling effects. There remains a lack of choice of therapies for managing the remodeling specifically in diabetic heart. Our findings provide insight into the novel mechanisms underlying the cardioprotective action of AGEs-formation inhibitors and have important implications for preventing the diabetes-related cardiac dysfunction and arrhythmias. 


\section{Acknowledgments}

We thank Shi-Han Weng, Shang-Yu Tsai, Yu-Ting Liu, Chia-Ling Chen, Yu-Ci Liao, Ting-Hsiang Chan, and Wei-Chun Chen for technical assistance.

\section{Authorship Contributions}

Participated in research design: Chang, Yeh.

Conducted experiments: Chang, Yeh, Chen, Ko, Pang, Lee.

Performed data analysis: Chang, Yeh, Lee.

Wrote or contributed to the writing of the manuscript: Chang.

\section{References}

Bers DM (2002) Cardiac excitation-contraction coupling. Nature 415:198-205. Bidasee KR, Nallani K, Yu Y, Cocklin RR, Zhang Y, Wang M, Dincer UD, and Besch HR, Jr (2003) Chronic diabetes increases advanced glycation end products on cardiac ryanodine receptors/calcium-release channels. Diabetes 52:1825-1836.

Bidasee KR, Zhang Y, Shao CH, Wang M, Patel KP, Dincer UD, and Besch HR, Jr (2004) Diabetes increases formation of advanced glycation end products on Sarco(endo)plasmic reticulum $\mathrm{Ca}^{2+}$-ATPase. Diabetes 53:463-473.

Bodiga VL, Eda SR, and Bodiga S (2014) Advanced glycation end products: role in pathology of diabetic cardiomyopathy. Heart Fail Rev 19:49-63.

Brownlee M (2001) Biochemistry and molecular cell biology of diabetic complications. Nature 414:813-820.

Brownlee M, Vlassara H, Kooney A, Ulrich P, and Cerami A (1986) Aminoguanidine prevents diabetes-induced arterial wall protein cross-linking. Science 232 $1629-1632$.

Bugger H and Abel ED (2014) Molecular mechanisms of diabetic cardiomyopathy. Diabetologia 57:660-671.

Chang GJ, Yeh YH, Lin TP, Chang CJ, and Chen WJ (2014) Electromechanical and atrial and ventricular antiarrhythmic actions of CIJ-3-2F, a novel benzylfuroquinoline vasodilator in rat heart. $\mathrm{Br} J$ Pharmacol 171:3918-3937.

Choi KM, Zhong Y, Hoit BD, Grupp IL, Hahn H, Dilly KW, Guatimosim S, Lederer WJ, and Matlib MA (2002) Defective intracellular $\mathrm{Ca}(2+)$ signaling contributes to cardiomyopathy in type 1 diabetic rats. Am J Physiol Heart Circ Physiol 283 H1398-H1408.

Corbett JA, Tilton RG, Chang K, Hasan KS, Ido Y, Wang JL, Sweetland MA, Lancaster JR, Jr, Williamson JR, and McDaniel ML (1992) Aminoguanidine, a novel inhibitor of nitric oxide formation, prevents diabetic vascular dysfunction. Diabetes 41:552-556.

Giardino I, Fard AK, Hatchell DL, and Brownlee M (1998) Aminoguanidine inhibits reactive oxygen species formation, lipid peroxidation, and oxidant-induced apoptosis. Diabetes 47:1114-1120.

Hegab Z, Mohamed TMA, Stafford N, Mamas M, Cartwright EJ, and Oceandy D (2017) Advanced glycation end products reduce the calcium transient in cardiomyocytes by increasing production of reactive oxygen species and nitric oxide. FEBS Open Bio 7:1672-1685.

Howarth FC, Jacobson M, Qureshi MA, Shafiullah M, Hameed RS, Zilahi E, Al Haj A, Nowotny N, and Adeghate E (2009) Altered gene expression may underlie prolonged duration of the QT interval and ventricular action potential in streptozotocin-induced diabetic rat heart. Mol Cell Biochem 328:57-65.

Huynh K, Bernardo BC, McMullen JR, and Ritchie RH (2014) Diabetic cardiomyopathy: mechanisms and new treatment strategies targeting antioxidant signaling pathways. Pharmacol Ther 142:375-415.

Ishikawa T, Kajiwara H, and Kurihara S (1999) Alterations in contractile properties and $\mathrm{Ca}^{2+}$ handling in streptozotocin-induced diabetic rat myocardium. $\mathrm{Am} J$ Physiol 277:H2185-H2194.

Jourdon P and Feuvray D (1993) Calcium and potassium currents in ventricular myocytes isolated from diabetic rats. J Physiol 470:411-429.

Kato T, Yamashita T, Sekiguchi A, Sagara K, Takamura M, Takata S, Kaneko S, Aizawa T, and Fu LT (2006) What are arrhythmogenic substrates in diabetic rat atria? J Cardiovasc Electrophysiol 17:890-894.

Lacombe VA, Viatchenko-Karpinski S, Terentyev D, Sridhar A, Emani S, Bonagura JD, Feldman DS, Györke S, and Carnes CA (2007) Mechanisms of impaired calcium handling underlying subclinical diastolic dysfunction in diabetes. Am $J$ Physiol Regul Integr Comp Physiol 293:R1787-R1797.

$\mathrm{Li} \mathrm{X}, \mathrm{Xu} \mathrm{Z}, \mathrm{Li} \mathrm{S}$, and Rozanski GJ (2005) Redox regulation of Ito remodeling in diabetic rat heart. Am J Physiol Heart Circ Physiol 288:H1417-H1424.

Ma H, Li SY, Xu P, Babcock SA, Dolence EK, Brownlee M, Li J, and Ren J (2009) Advanced glycation endproduct (AGE) accumulation and AGE receptor (RAGE) up-regulation contribute to the onset of diabetic cardiomyopathy. J Cell Mol Med 13 (8B):1751-1764.

Mordenti J (1986) Man versus beast: pharmacokinetic scaling in mammals. J Pharm Sci 75:1028-1040.
Movahed MR, Hashemzadeh M, and Jamal MM (2005) Diabetes mellitus is a strong, independent risk for atrial fibrillation and flutter in addition to other cardiovascular disease. Int $J$ Cardiol 105:315-318.

National Research Council (NRC) (2011) Guide for the Care and Use of Laboratory Animals. 8th ed. National Academies Press, Washington, DC, http://grants.nih.gov/ grants/olaw/Guide-for-the-care-and-use-of-laboratory-animals.pdf.

Norton GR, Candy G, and Woodiwiss AJ (1996) Aminoguanidine prevents the decreased myocardial compliance produced by streptozotocin-induced diabetes mellitus in rats. Circulation 93:1905-1912.

Pacher P, Ungvári Z, Nánási PP, and Kecskeméti V (1999) Electrophysiological changes in rat ventricular and atrial myocardium at different stages of experimental diabetes. Acta Physiol Scand 166:7-13.

Petrova R, Yamamoto Y, Muraki K, Yonekura H, Sakurai S, Watanabe T, Li H, Takeuchi M, Makita Z, Kato I, et al. (2002) Advanced glycation endproduct-induced calcium handling impairment in mouse cardiac myocytes. J Mol Cell Cardiol 34: 1425-1431.

Qin D, Huang B, Deng L, El-Adawi H, Ganguly K, Sowers JR, and El-Sherif N (2001) Downregulation of $\mathrm{K}(+)$ channel genes expression in type I diabetic cardiomyopathy. Biochem Biophys Res Commun 283:549-553.

Shao CH, Rozanski GJ, Patel KP, and Bidasee KR (2007) Dyssynchronous (nonuniform) $\mathrm{Ca}^{2+}$ release in myocytes from streptozotocin-induced diabetic rats. $J \mathrm{Mol}$ Cell Cardiol 42:234-246.

Shi FH, Cheng YS, Dai DZ, Peng HJ, Cong XD, and Dai Y (2013) Depressed calciumhandling proteins due to endoplasmic reticulum stress and apoptosis in the diabetic heart are attenuated by argirein. Naunyn Schmiedebergs Arch Pharmacol 386:521-531.

Sorrentino A, Borghetti G, Zhou Y, Cannata A, Meo M, Signore S, Anversa P, Leri A, Goichberg P, Qanud K, et al. (2017) Hyperglycemia induces defective $\mathrm{Ca}^{2+}$ homeostasis in cardiomyocytes. Am J Physiol Heart Circ Physiol 312:H150-H161.

Stadler K, Jenei V, Somogyi A, and Jakus J (2005) Beneficial effects of aminoguanidine on the cardiovascular system of diabetic rats. Diabetes Metab Res Rev 21:189-196.

Sumi D and Ignarro LJ (2004) Regulation of inducible nitric oxide synthase expression in advanced glycation end product-stimulated raw 264.7 cells: the role of heme oxygenase-1 and endogenous nitric oxide. Diabetes 53:1841-1850.

Veglio M, Chinaglia A, and Cavallo-Perin P (2004) QT interval, cardiovascular risk factors and risk of death in diabetes. $J$ Endocrinol Invest 27:175-181.

Wang DW, Kiyosue T, Shigematsu S, and Arita M (1995) Abnormalities of $\mathrm{K}^{+}$and $\mathrm{Ca}^{2+}$ currents in ventricular myocytes from rats with chronic diabetes. $\mathrm{Am} J$ Physiol 269:H1288-H1296.

Watanabe M, Yokoshiki H, Mitsuyama H, Mizukami K, Ono T, and Tsutsui H (2012) Conduction and refractory disorders in the diabetic atrium. Am $J$ Physiol Heart Circ Physiol 303:H86-H95.

Willemsen S, Hartog JW, van Veldhuisen DJ, van der Meer P, Roze JF, Jaarsma T, Schalkwijk C, van der Horst IC, Hillege HL, and Voors AA (2012) The role of advanced glycation end-products and their receptor on outcome in heart failure patients with preserved and reduced ejection fraction. Am Heart $J$ 164:742-749.e3.

Wu MS, Liang JT, Lin YD, Wu ET, Tseng YZ, and Chang KC (2008) Aminoguanidine prevents the impairment of cardiac pumping mechanics in rats with streptozotocin and nicotinamide-induced type 2 diabetes. Br J Pharmacol 154:758-764.

Yamagishi S, Nakamura K, Matsui T, Ueda S, Noda Y, and Imaizumi T (2008) Inhibitors of advanced glycation end products (AGEs): potential utility for the treatment of cardiovascular disease. Cardiovasc Ther 26:50-58.

Yang Z, Shen W, Rottman JN, Wikswo JP, and Murray KT (2005) Rapid stimulation causes electrical remodeling in cultured atrial myocytes. $J$ Mol Cell Cardiol 38: 299-308

Yaras N, Ugur M, Ozdemir S, Gurdal H, Purali N, Lacampagne A, Vassort G, and Turan B (2005) Effects of diabetes on ryanodine receptor Ca release channel (RyR2) and $\mathrm{Ca}^{2+}$ homeostasis in rat heart. Diabetes 54:3082-3088.

Yeh YH, Hsu LA, Chen YH, Kuo CT, Chang GJ, and Chen WJ (2016) Protective role of heme oxygenase-1 in atrial remodeling. Basic Res Cardiol 111:58.

Yuan Q, Zhan L, Zhou QY, Zhang LL, Chen XM, Hu XM, and Yuan XC (2015) SIRT2 regulates microtubule stabilization in diabetic cardiomyopathy. Eur J Pharmacol 764:554-561.

Zhang L, Cannell MB, Phillips AR, Cooper GJ, and Ward ML (2008) Altered calcium homeostasis does not explain the contractile deficit of diabetic cardiomyopathy. Diabetes 57:2158-2166.

Zhong Y, Ahmed S, Grupp IL, and Matlib MA (2001) Altered SR protein expression associated with contractile dysfunction in diabetic rat hearts. Am J Physiol Heart Circ Physiol 281:H1137-H1147.

Address correspondence to: Dr. Gwo-Jyh Chang, Graduate Institute of Clinical Medicinal Sciences, College of Medicine, Chang Gung University, 259, Wen-Hwa 1st Road, Kwei-Shan, Tao-Yuan, Taiwan 33302. E-mail: gjchang@ mail.cgu.edu.tw 